\title{
Numerical solution of a fractal-fractional order chaotic circuit system
}

\author{
Muhammad Altaf Khan ${ }^{a, *}$, A. Atangana ${ }^{a, b}$, Taseer Muhammad ${ }^{c}$, and Ebraheem Alzahrani ${ }^{d}$ \\ ${ }^{a}$ Institute for Groundwater Studies, University of the Free State, Bloemfontein 9301, South Africa. \\ *e-mail: altafdir@gmail.com \\ ${ }^{b}$ Department of Medical Research China Medical University Hospital, \\ China Medical University, Taichung, Taiwan. \\ ${ }^{c}$ Department of Mathematics, College of Sciences, \\ King Khalid University, Abha 61413, Saudi Arabia. \\ ${ }^{d}$ Department of Mathematics, Faculty of Science, King Abdulaziz University, \\ P.O. Box 80203, Jeddah 21589, Saudi Arabia.
}

Received 19 March 2021; accepted 22 April 2021

The dynamical system has an important research area and, due to its wide applications, many researchers and scientists are working to develop new models and techniques for their solution. In this work, we present in this work the dynamics of a chaotic model in the presence of newly introduced fractal-fractional operators. The model is formulated initially in ordinary differential equations, and then we utilize the fractal-fractional (FF) in power law, exponential, and Mittag-Leffler to generalize the model. For each fractal-fractional order model, we briefly study its numerical solution via the numerical algorithm. We present some graphical results with arbitrary order of fractal and fractional orders, and present that these operators provide different chaotic attractors for different fractal and fractional order values. The graphical results demonstrate the effectiveness of the fractal-fractional operators.

Keywords: Fractal-fractional operators; chaotic model; numerical results.

PACS: 05.45.-a; 05.40.-a; 02.60.Cb

DOI: https://doi.org/10.31349/RevMexFis.67.051401

\section{Introduction}

The fractional differential equation (FDE) and the fractional calculus (FC) are now the most growing research areas and receiving good feedback from scientists and authors working in modeling real-life problems in the scientific and engineering fields. It is well-known that the fractional differential equation has many applications in scientific and engineering sectors. In particular, the dynamical systems and the chaos theory are also getting much attention from the researchers due to their wide applications in many fields such as autonomous mobile robots, chaotic communication, image watermarking, and circuits, etcetera [1-3]. Many researchers studied the chaotic and other models in fractional operators see for example [4-15]. These operators in power law, exponential decay, and Mittag-Leffler kernel were utilized successfully to present the various models of practical purpose. For example, the authors considered in Ref. [4] the chaotic financial model and its control strategy. The time delay chaotic model for the circuit and its synchronization has been investigated in Ref. [5]. The fractal-fractional (FF) operators introduced recently has been getting an impressive response from researchers. Using the idea of FF and its application to the existing chaotic models in the literature are studied in Ref. [6]. The new chaotic system in fractional derivative and its comparative study is suggested in Ref. [7], while the application of fractional calculus to African tortoise and image processing has been discussed in Ref. [8,9]. The application of fractional derivative to zoonotic disease [10], application to dengue epidemic, see [11], tuberculosis, see [12-14], and the Chua model [15] has been investigated in arbitrary derivative.

Mostly the problems formulated in scientific and engineering areas are of high nonlinear nature, and its exact solution is not possible. The solution of a fractional differentiable equation is also very complicated due to its order and difficult to solve analytically, and thus the researchers always looking to have their solution numerically. Fractional differential equation and its application to nonlinear problems of different fields with novel numerical investigations have been studied in Refs. $[10,16,17]$ and the references therein. Nowadays the researchers from different areas developing mathematical models in fractional calculus due to its applications. One of the interesting applications is the best fitting to the real cases for an epidemic disease and another real data setting that come from different areas of engineering and sciences. The memory and the heredity properties are attached to the fractional operators and can best determine the dynamics of dynamical systems and other related areas problems efficiently than the classical orders. It is the advantage of fractional calculus that can determine well the analysis between any two suggested points. Some recent work related to fractional operators and their applications can be seen in Refs. [18-20]. In this work, the authors focused on studying the circuit system, fractional-order capacitor, and sound encryption system using arbitrary order derivatives. The fractal order deriva- 
tive and the fractional-order, as well as their combinations for the solution of physical problems have been suggested in Refs. [21-26]. The chaotic Shinriki's oscillator fractional model has been investigated by the authors in Ref. [21]. In Ref. [22], the authors studied the periodicity and multiple attractors for Villis model. The idea of FF has been considered to study influenza and malaria model in Refs. [23,24]. The fractal-fractional operators have been applied effectively for the related problems, see [25-27], where the authors obtain results for new chaotic attractors, the study of the Hepatitis C model, and for system identification. Some more interesting work, where the authors proposed the fractional derivatives as an application to the scientific problems, see [28-35]. For example, the author in Ref. [28] used the Laplace transform for getting the solution of a fractional telegraph equation. The formulation of the tumor fractional model using nonsingular kernel has been studied in Ref. [29]. In Ref. [30], the authors studied the solution of the fifth-order evolution equation in a fractional derivative. Using the Haar wavelet method, the authors in Ref. [31] obtained the solution of the Lotka-Volterra model related to population in a fractional derivative. Using Yang-Abdel-Aty-Cattani fractional operator, the authors discussed the solution of heat equations in Ref. [32]. The classical optical solution for the propagation model has been derived in Ref. [33]. The two interacting species model using numerical method is discussed in Ref. [34]. A fractional-order SEIR model using Genocchi has been studied in Ref. [35].

In this investigation, we considered the FF designed operators suggested in the sense of power law, exponential decay, and the Mittag-Leffler kernel. These suggested operators were found interesting from a researchers point of view and have been found reliable for chaotic order systems. Using these operators, many hidden attractors have been found which were not possible for ordinary fractional operators. It is clear that these operators attract various attractors by considering their different orders. Recently, fractal-fractional orders problems are formulated in Ref. $[6,36]$, etc. It is wellknown that the results shown in Ref. $[6,36]$ are the applications for a physical systems. Further, these operators were found best for data fitting; one can see [36]. Moreover, the results suggested in Refs. [6,36-38] demonstrate the combinations of these FF operators provide many insights about the dynamics, which can only be observed for the FF orders. With the above many useful applications of the FF operators, we give a chaotic model and present its analysis and provide a useful and effective scheme for its solution. We will find many useful numerical results by considering the combinations of the FF orders.

The work shown in this paper has been arranged sectionwise is as follows. We provide the details of the FF operators and model description in Secs. 2 and 3. Section 4 presents the novel solution technique for the FF model in Caputo, CF, and $\mathrm{AB}$ operators. We discussed the simulation results in Secs. 5 and 6 , we summarized the finding.

\section{Fundamental of FF operators}

The present section determines the necessary results associated with fractal-fractional differential and their corresponding integral $[6,36]$.

Definition 1. A function $g(t)$ with fractal order $\beta$ and fractional order $\alpha$ is continuous and differentiable over $\left(m_{1}, m_{2}\right)$, then, in a power-law sense, the following is presented:

$$
{ }^{F F P} D_{0, t}^{\alpha, \beta}(g(t))=\frac{1}{\Gamma(k-\alpha)} \frac{d}{d t^{\beta}} \int_{0}^{t}(t-\delta)^{m-\alpha-1} g(\delta) d \delta,
$$

with $k-1<\alpha, \beta \leq k \in \mathbb{N}$ and $d g(t) / d t^{\beta}=$ $\lim _{t_{1} \rightarrow t}\left(g\left(t_{1}\right)-g(t) / t_{1}^{\beta}-t^{\beta}\right)$.

Definition 2. A function $g(t)$ with fractal order $\beta$ and fractional order $\alpha$ is continuous and differentiable over $\left(m_{1}, m_{2}\right)$, then, in exponential decay kernel, the following is shown:

$$
\begin{aligned}
{ }^{F F E} D_{0, t}^{\alpha, \beta}(g(t)) & =\frac{M(\alpha)}{1-\alpha} \frac{d}{d t^{\beta}} \\
& \times \int_{0}^{t} \exp \left(-\frac{\alpha}{1-\alpha}(t-\delta)\right) g(\delta) d \delta,
\end{aligned}
$$

with $M(0)=M(1)=1$ and for positive $\alpha$ with $\beta \leq m \in \mathbb{N}$. Definition 3. A function $g(t)$ with fractal and fractional or$\operatorname{der} \beta$ and $\alpha$ respectively, is continuous and differentiable over $\left(m_{1}, m_{2}\right)$, then, the Mittag-Leffler kernel follows the formulate given by:

$$
\begin{aligned}
{ }^{F F M} D_{0, t}^{\alpha, \beta} & (g(t))=\frac{A B(\alpha)}{1-\alpha} \frac{d}{d t^{\beta}} \\
& \times \int_{0}^{t} E_{\alpha}\left(-\frac{\alpha}{1-\alpha}(t-\delta)^{\alpha}\right) g(\delta) d \delta,
\end{aligned}
$$

with $\alpha>0, \beta \leq 1 \in \mathbb{N}$ and $A B(\alpha)=1-\alpha+(\alpha / \Gamma(\alpha))$.

Definition 4. A function $g(t)$ continuous over $\left(m_{1}, m_{2}\right)$, with fractal order $\beta$ and fractional order $\alpha$ then, for the power-law kernel, we have the following integral:

$$
{ }_{F F P} J_{0, t}^{\alpha}(g(t))=\frac{\beta}{\Gamma(\alpha)} \int_{0}^{t}(t-\delta)^{\alpha-1} \delta^{\beta-1} g(\delta) d \delta .
$$

Definition 5. A function $g(t)$ continuous over $\left(m_{1}, m_{2}\right)$, with fractal order $\beta$ and fractional order $\alpha$ then, the FF integral exponentially decaying kernel for $f(t)$ is described by the following:

$$
\begin{aligned}
F F E J_{0, t}^{\alpha}(g(t)) & =\frac{\alpha \beta}{M(\alpha)} \int_{0}^{t} \delta^{\alpha-1} g(\delta) d \delta \\
& +\frac{\beta(1-\alpha) t^{\beta-1} g(t)}{M(\alpha)} .
\end{aligned}
$$


Definition 6. A function $z(t)$ continuous on $(a, b)$, with fractal order $\beta$ and fractional order $\alpha$ then, for the Mittag-Leffler kernel, the following integral is suggested:

$$
\begin{aligned}
F F M J_{0, t}^{\alpha, \beta}(g(t)) & =\frac{\alpha \beta}{A B(\alpha)} \int_{0}^{t} \delta^{\beta-1}(t-\delta)^{\alpha-1} g(\delta) d \delta \\
& +\frac{\beta(1-\alpha) t^{\beta-1} g(t)}{A B(\alpha)} .
\end{aligned}
$$

Lemma 1. The following result holds for fractional and fractal order $\alpha$, and $\beta$, respectively,

$$
{ }_{0}^{F F} J_{t}^{\alpha, \beta} g(t)=\frac{\beta}{\Gamma(\alpha)} \int_{0}^{t} t^{\beta-1}(t-\delta)^{\alpha-1} g(\delta) d \delta .
$$

Proof. Consider

$$
\begin{aligned}
{ }_{0}^{C} D_{t}^{\alpha, \beta} g(t) & =u(t), \\
{ }_{0}^{C} D_{t}^{\alpha} g(t) & =\beta t^{\beta-1} u(t) .
\end{aligned}
$$

Riemann-Liouville integral gives the following,

$$
\begin{aligned}
& g(t)=\frac{1}{\Gamma(\alpha)} \int_{0}^{t} \beta t^{\beta-1}(t-\delta)^{\alpha-1} u(\delta) d \delta, \\
& g(t)=\frac{\beta}{\Gamma(\alpha)} \int_{0}^{t} t^{\beta-1}(t-\delta)^{\alpha-1} u(\delta) d \delta .
\end{aligned}
$$

Therefore,

$$
{ }_{0}^{F F} J_{t}^{\alpha, \beta} g(t)=\frac{\beta}{\Gamma(\alpha)} \int t 0 t^{\beta-1}(t-\delta)^{\alpha-1} g(\delta) d \delta
$$

Lemma 2. The following result holds for fractional and fractal order $\alpha$ and $\beta$, respectively,

$$
{ }_{0}^{F F P} D_{t}^{\alpha, \beta} g(t)=\frac{1}{\Gamma(1-\alpha)} \frac{d}{d t^{\beta}} \int_{0}^{t}(t-\delta)^{-\alpha} g(\delta) d \delta .
$$

Proof. In order to show this result given by

$$
{ }_{0}^{F F P} D_{t}^{\alpha, \beta} g(t)=\frac{1}{\Gamma(1-\alpha)} \frac{d}{d t^{\beta}} \int_{0}^{t}(t-\delta)^{-\alpha} g(\delta) d \delta .
$$

Since

$$
\int_{0}^{t}(t-\delta)^{-\alpha} g(\delta) d \delta
$$

is differentiable, so, we can use

$$
F(t)=\int_{0}^{t}(t-\delta)^{-\alpha} g(\delta) d \delta
$$

So,

$$
\begin{aligned}
{ }_{0}^{F F P} D_{t}^{\alpha, \beta} g(t) & =\frac{1}{\Gamma(1-\alpha)} \frac{d}{d t^{\beta}} F(t), \\
& =\frac{1}{\Gamma(1-\alpha)} \lim _{t_{1} \rightarrow t} \frac{F\left(t_{1}\right)-F(t)}{t_{1}^{\beta}-t^{\beta}}, \\
& =\frac{1}{\Gamma(1-\alpha)} \lim _{t_{1} \rightarrow t} \frac{F\left(t_{1}\right)-F(t)}{t_{1}-t} \frac{t_{1}-t}{t_{1}^{\beta}-t^{\beta}}, \\
& =\frac{1}{\Gamma(1-\alpha)} F^{\prime}(t) \frac{1}{\beta t^{\beta-1}} .
\end{aligned}
$$

\section{The chaotic model}

We consider a model which chaotic and has been presented in Ref. [39]. The model has three equations, and its differential equations form considered in Ref. [39] is shown below:

$$
\left\{\begin{array}{l}
\frac{d x(t)}{d t}=y, \\
\frac{d y(t)}{d t}=y z-x, \\
\frac{d z(t)}{d t}=1-y^{2},
\end{array}\right.
$$

where $x(t), y(t)$ and $z(t)$ represent the state variables and become chaotic for the initial values of the variables $x(0)=0$, $y(0)=1$ and $z(0)=0$. The fractal-fractional representation of the model (7) is given by:

$$
\left\{\begin{array}{l}
{ }_{F F P} D_{0, t}^{\alpha, \beta}(x(t))=y, \\
{ }_{F F P} D_{0, t}^{\alpha, \beta}(y(t))=y z-x, \\
{ }_{F F P} D_{0, t}^{\alpha, \beta}(z(t))=1-y^{2},
\end{array}\right.
$$

where $\alpha$ represents the fractional order while $\beta$ is fractal order. No equilibria exist for the model (8). Further, in the following, we are going to formulate the problem above using FF operators and present the numerical scheme for each case.

\section{Numerical procedure for the chaotic model}

This section presents briefly the procedure to handle the model formulated in FF with fractional operators sense, $\mathrm{Ca}-$ puto, $\mathrm{CF}$, and $\mathrm{AB}$. One by one, we apply the fractal operator to the model described above (8). 


\subsection{Numerical scheme for FF-Caputo model}

We start the model to provide a numerical algorithm by considering the model in power-law case. To start the algorithm, we need first the write the model (8) in Volterra representations, because the fractional integral is differentiable, so in the Riemann-Liouville (RL) sense, we have,

$$
{ }_{F F P} D_{0, t}^{\alpha, \beta} f(t)=\frac{1}{\Gamma(1-\alpha)} \frac{d}{d t} \int_{0}^{t}(t-\delta)^{-\alpha} f(\delta) d \delta \frac{1}{\beta t^{\beta-1}},
$$

we present the results in the following,

$$
\begin{aligned}
{ }^{R L} D_{0, t}^{\alpha}(x(t)) & =\beta t^{\beta-1}[y], \\
{ }^{R L} D_{0, t}^{\alpha}(y(t)) & =\beta t^{\beta-1}[y z-x], \\
{ }^{R L} D_{0, t}^{\alpha}(z(t)) & =\beta t^{\beta-1}\left[1-y^{2}\right] .
\end{aligned}
$$

Consider now replacing the operator shown by Riemann-Liouville(RL) in the above system with the Caputo operator to get the initial conditions in the integer case. Further, we get the result by using RL fractional integral:

$$
\begin{aligned}
& x(t)=x(0)+\frac{\beta}{\Gamma(\alpha)} \int_{0}^{t} \delta^{\beta-1}(t-\delta)^{\alpha-1} f_{1}(x, y, z, \delta) d \delta, \\
& y(t)=y(0)+\frac{\beta}{\Gamma(\alpha)} \int_{0}^{t} \delta^{\beta-1}(t-\delta)^{\alpha-1} f_{2}(x, y, z, \delta) d \delta, \\
& z(t)=z(0)+\frac{\beta}{\Gamma(\alpha)} \int_{0}^{t} \delta^{\beta-1}(t-\delta)^{\alpha-1} f_{3}(x, y, z, \delta) d \delta,
\end{aligned}
$$

where

$$
f_{1}(x, y, z, \delta)=y, \quad f_{2}(x, y, z, \delta)=y z-x, \quad f_{3}(x, y, z, \delta)=1-y^{2} .
$$

For $t=t_{n+1}$, we have the following,

$$
\begin{aligned}
& x^{n+1}=x^{0}+\frac{\beta}{\Gamma(\alpha)} \int_{0}^{t_{n+1}} \delta^{\beta-1}\left(t_{n+1}-\delta\right)^{\alpha-1} f_{1}(x, y, z, \delta) d \delta, \\
& y^{n+1}=y^{0}+\frac{\beta}{\Gamma(\alpha)} \int_{0}^{t_{n+1}} \delta^{\beta-1}\left(t_{n+1}-\delta\right)^{\alpha-1} f_{2}(x, y, z, \delta) d \delta, \\
& z^{n+1}=z^{0}+\frac{\beta}{\Gamma(\alpha)} \int_{0}^{t_{n+1}} \delta^{\beta-1}\left(t_{n+1}-\delta\right)^{\alpha-1} f_{3}(x, y, z, \delta) d \delta .
\end{aligned}
$$

Simplifying the above results further, we get

$$
\begin{aligned}
& x^{n+1}=x^{0}+\frac{\beta}{\Gamma(\alpha)} \sum_{j=0}^{n} \int_{t_{j}}^{t_{j+1}} \delta^{\beta-1}\left(t_{n+1}-\delta\right)^{\alpha-1} f_{1}(x, y, z, \delta) d \delta, \\
& y^{n+1}=y^{0}+\frac{\beta}{\Gamma(\alpha)} \sum_{j=0}^{n} \int_{t_{j}}^{t_{j+1}} \delta^{\beta-1}\left(t_{n+1}-\delta\right)^{\alpha-1} f_{2}(x, y, z, \delta) d \delta, \\
& z^{n+1}=z^{0}+\frac{\beta}{\Gamma(\alpha)} \sum_{j=0}^{n} \int_{t_{j}}^{t_{j+1}} \delta^{\beta-1}\left(t_{n+1}-\delta\right)^{\alpha-1} f_{3}(x, y, z, \delta) d \delta,
\end{aligned}
$$


where $t(0)=0$. The functions $\delta^{\beta-1} f_{i}(x, y, z, \delta)$ for $i=1,2,3$ shown in Eq. (13) are approximated in the interval $\left[t_{j}, t_{j+1}\right]$ using the Lagrangian interpolation concept, and getting the following,

$$
\begin{aligned}
L_{j}(\delta) & =\frac{\delta-t_{j-1}}{t_{j}-t_{j-1}} t_{j}^{\beta-1} f_{1}\left(x^{j}, y^{j}, z^{j}, t_{j}\right)-\frac{\delta-t_{j}}{t_{j}-t_{j-1}} t_{j-1}^{\beta-1} f_{1}\left(x^{j-1}, y^{j-1}, z^{j-1}, t_{j-1}\right), \\
M_{j}(\delta) & =\frac{\delta-t_{j-1}}{t_{j}-t_{j-1}} t_{j}^{\beta-1} f_{2}\left(x^{j}, y^{j}, z^{j}, t_{j}\right)-\frac{\delta-t_{j}}{t_{j}-t_{j-1}} t_{j-1}^{\beta-1} f_{2}\left(x^{j-1}, y^{j-1}, z^{j-1}, t_{j-1}\right), \\
N_{j}(\delta) & =\frac{\delta-t_{j-1}}{t_{j}-t_{j-1}} t_{j}^{\beta-1} f_{3}\left(x^{j}, y^{j}, z^{j}, t_{j}\right)-\frac{\delta-t_{j}}{t_{j}-t_{j-1}} t_{j-1}^{\beta-1} f_{3}\left(x^{j-1}, y^{j-1}, z^{j-1}, t_{j-1}\right) .
\end{aligned}
$$

Using the results shown in (14), we have the below system,

$$
\begin{aligned}
& x^{n+1}=x^{0}+\frac{\beta}{\Gamma(\alpha)} \sum_{j=0}^{n} \int_{t_{j}}^{t_{j+1}} \delta^{\beta-1}\left(t_{n+1}-\delta\right)^{\alpha-1} L_{j}(\delta) d \delta, \\
& y^{n+1}=y^{0}+\frac{\beta}{\Gamma(\alpha)} \sum_{j=0}^{n} \int_{t_{j}}^{t_{j+1}} \delta^{\beta-1}\left(t_{n+1}-\delta\right)^{\rho-1} M_{j}(\delta) d \delta, \\
& z^{n+1}=z^{0}+\frac{\beta}{\Gamma(\alpha)} \sum_{j=0}^{n} \int_{t_{j}}^{t_{j+1}} \delta^{\beta-1}\left(t_{n+1}-\delta\right)^{\rho-1} N_{j}(\delta) d \delta .
\end{aligned}
$$

Further solution of system (15) leads to the following,

$$
\begin{aligned}
& x^{n+1}=x^{0}+\frac{\beta h^{\alpha}}{\Gamma(\alpha+2)} \sum_{j=1}^{n}\left[t_{j}^{\beta-1} f_{1}\left(x^{j}, y^{j}, z^{j}, t_{j}\right) a_{n, j}-t_{j-1}^{\beta-1} f_{1}\left(x^{j-1}, y^{j-1}, z^{j-1}, t_{j-1}\right) b_{n, j}\right], \\
& y^{n+1}=y^{0}+\frac{\beta h^{\alpha}}{\Gamma(\alpha+2)} \sum_{j=1}^{n}\left[t_{j}^{\beta-1} f_{2}\left(x^{j}, y^{j}, z^{j}, t_{j}\right) a_{n, j}-t_{j-1}^{\beta-1} f_{2}\left(x^{j-1}, y^{j-1}, z^{j-1}, t_{j-1}\right) b_{n, j}\right], \\
& z^{n+1}=z^{0}+\frac{\beta h^{\alpha}}{\Gamma(\alpha+2)} \sum_{j=1}^{n}\left[t_{j}^{\beta-1} f_{3}\left(x^{j}, y^{j}, z^{j}, t_{j}\right) a_{n, j}-t_{j-1}^{\beta-1} f_{3}\left(x^{j-1}, y^{j-1}, z^{j-1}, t_{j-1}\right) b_{n, j}\right],
\end{aligned}
$$

where

$$
\begin{aligned}
& a_{n, j}=\left((n+1-j)^{\alpha}(n-j+2+\alpha)-(n-j)^{\alpha}(n-j+2+2 \alpha)\right), \\
& b_{n, j}=\left((n-j+1)^{\alpha+1}-(n-j)^{\alpha}(n-j+1+\alpha)\right),
\end{aligned}
$$

where $n=0$ to $N$ and $j=1$ to $n$.

\subsection{Numerical solution of FF-CF model}

Now, we consider the model in the FF-CF case and present in detail the numerical scheme. For this, we need to write the model in FF exponential decay form using the following structure:

$$
\begin{aligned}
& { }^{C F} D_{0, t}^{\alpha}(x(t))=\beta t^{\beta-1} f_{1}(x, y, z, t), \\
& { }^{C F} D_{0, t}^{\alpha}(y(t))=\beta t^{\beta-1} f_{2}(x, y, z, t), \\
& { }^{C F} D_{0, t}^{\alpha}(z(t))=\beta t^{\beta-1} f_{3}(x, y, z, t) .
\end{aligned}
$$




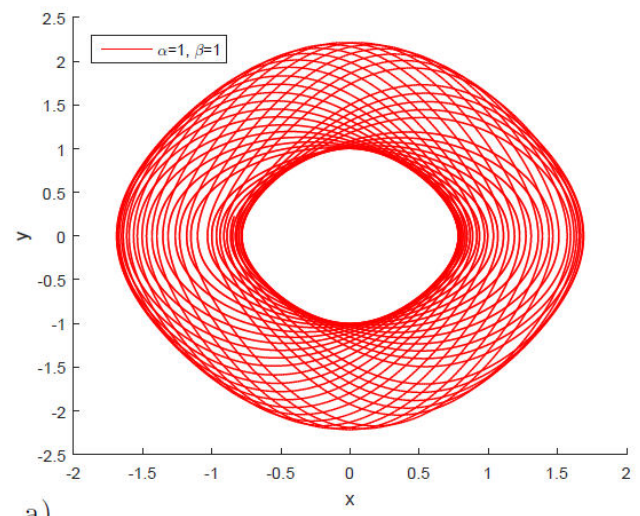

a)
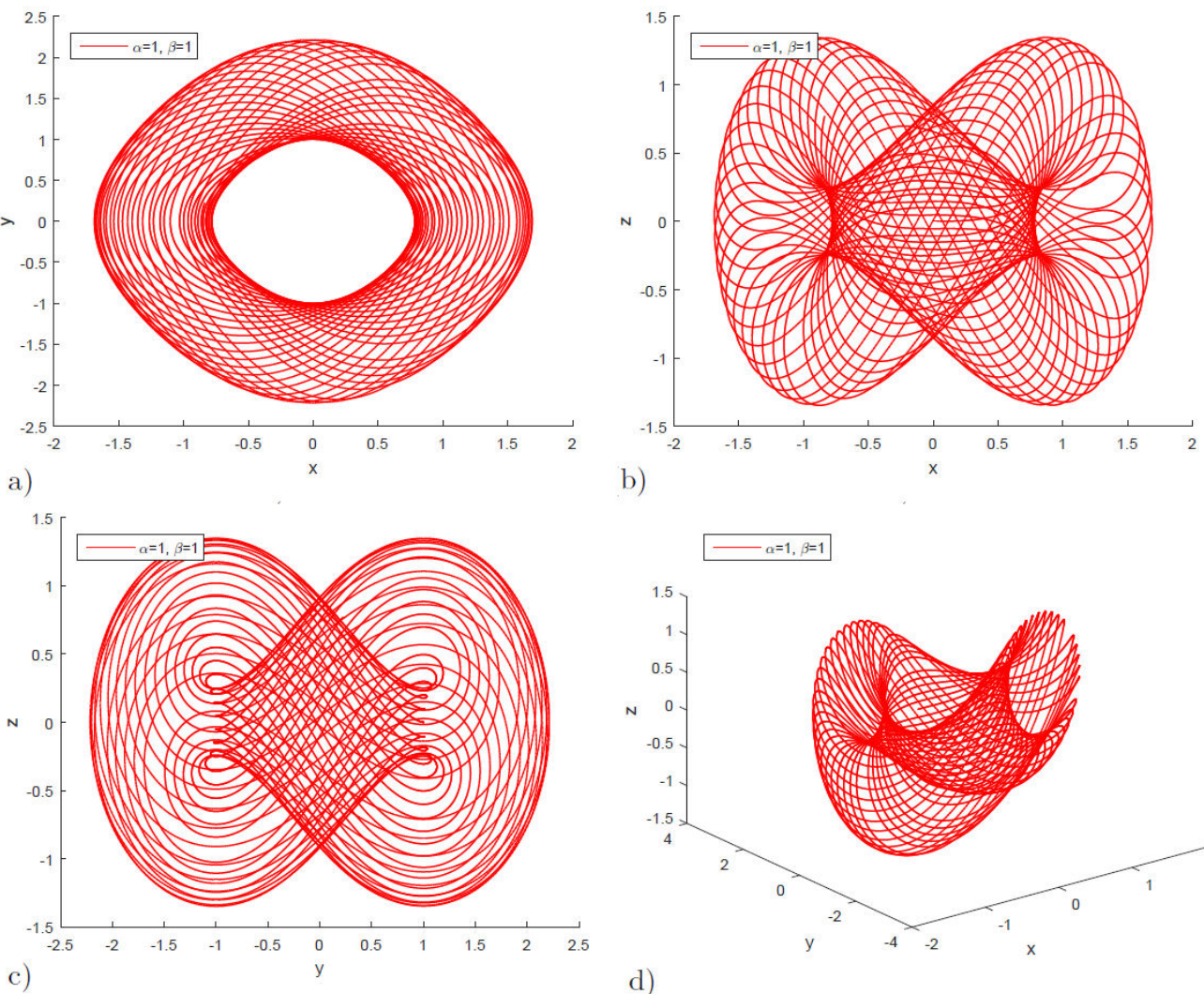

b)

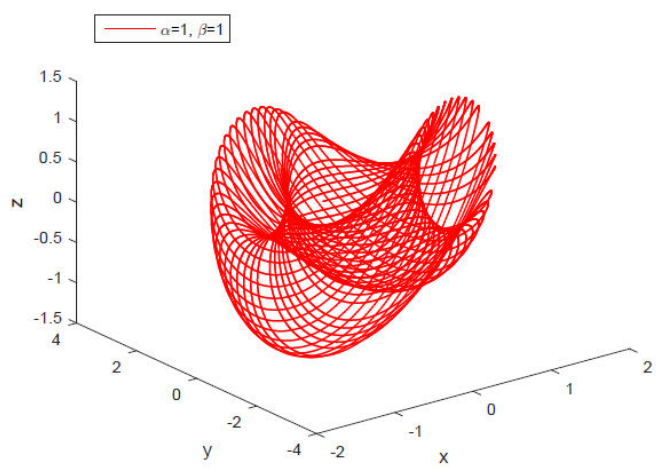

d)

FIGURE 1. FF-Caputo model results, when $\alpha=1, \beta=1$, where (a-d) show different phase planes.

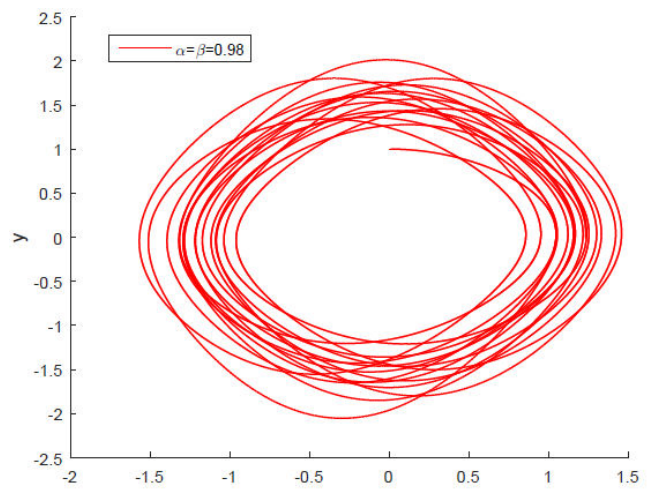

a)
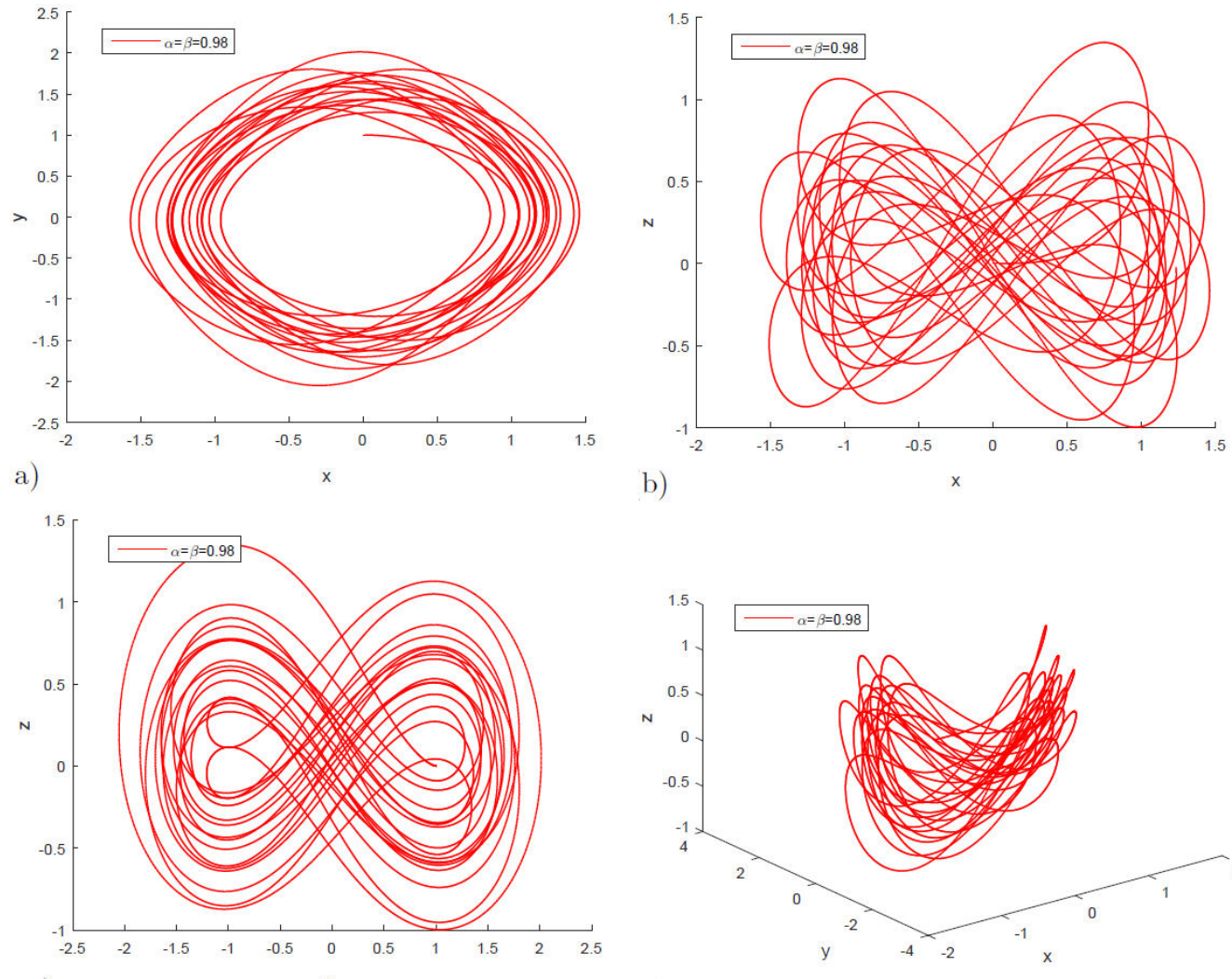

c)

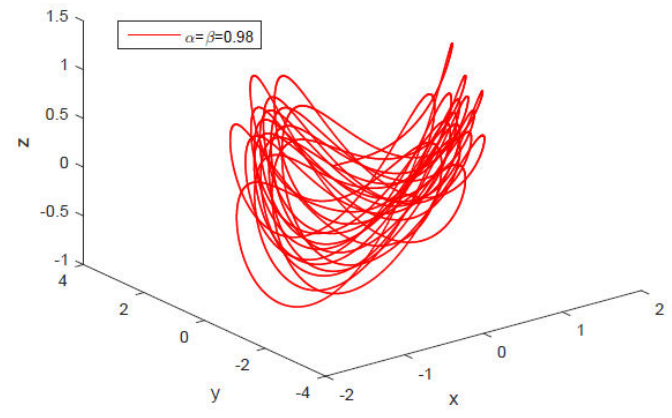

d)

FIGURE 2. FF-Caputo model results, when $\alpha=\beta=0.98$, where (a-d) show different phase planes. 

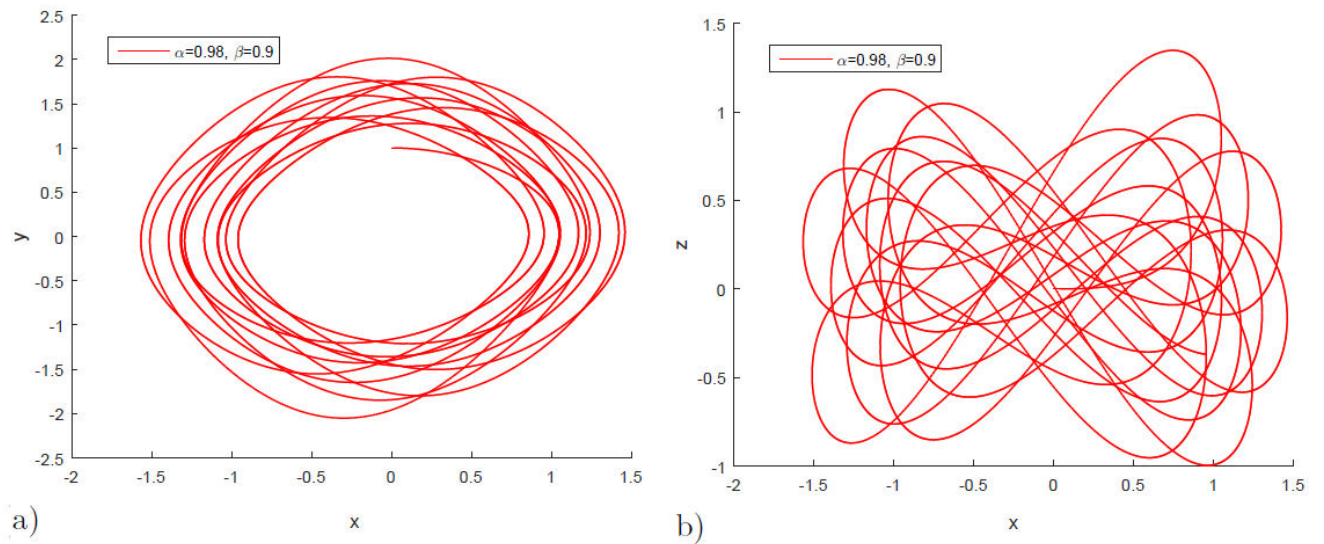

a)

$x$

b)

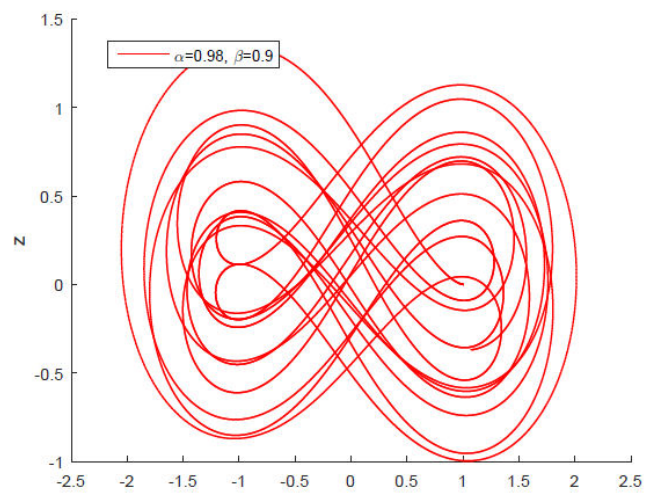

c)

y

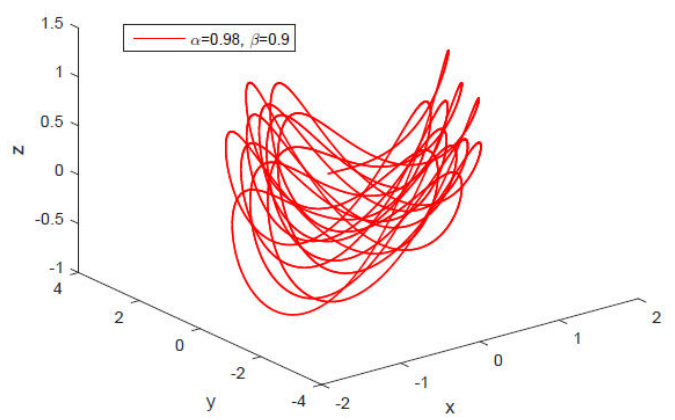

d)

FIGURE 3. FF-Caputo model results, when $\alpha=0.98, \beta=0.9$, where (a-d) show different phase planes.
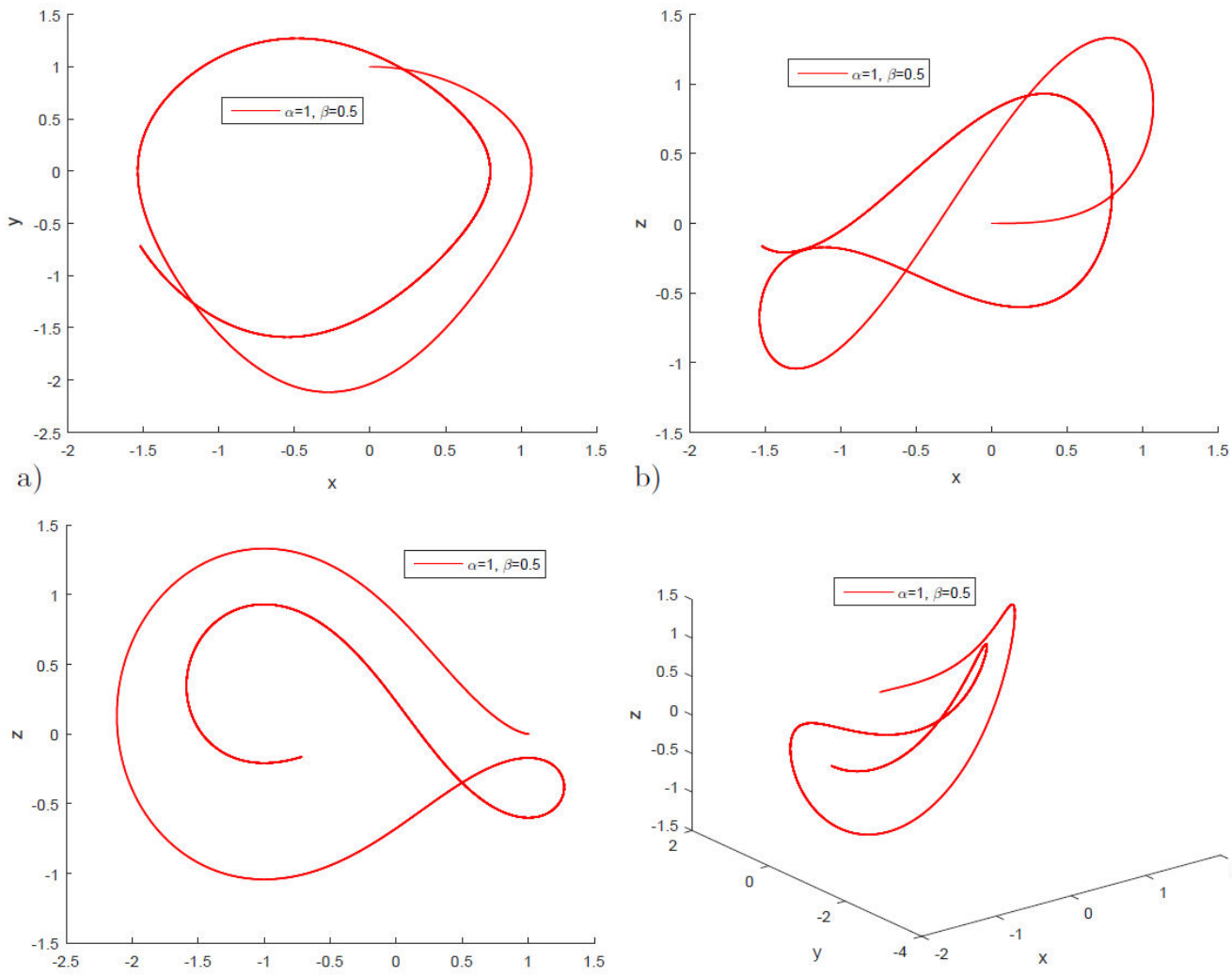

c)

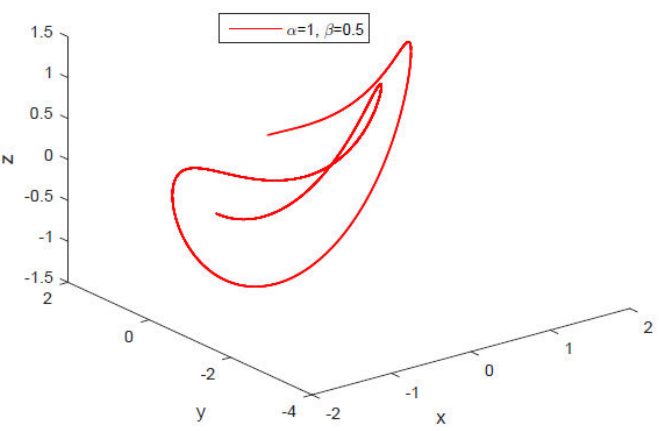

d)

FIGURE 4. FF-Caputo model results, when $\alpha=1, \beta=0.5$, where (a-d) show different phase planes. 
We obtain the following by using the Caputo-Fabrizio integral,

$$
\begin{aligned}
& x(t)=x^{0}+\frac{\beta t^{\beta-1}(1-\alpha)}{M(\alpha)} f_{1}(x, y, z, t)+\frac{\alpha \beta}{M(\alpha)} \int_{0}^{t} \delta^{\beta-1} f_{1}(x, y, z, \delta) d \delta, \\
& y(t)=y^{0}+\frac{\beta t^{\beta-1}(1-\alpha)}{M(\alpha)} f_{2}(x, y, z, t)+\frac{\alpha \beta}{M(\alpha)} \int_{0}^{t} \delta^{\beta-1} f_{2}(x, y, z, \delta) d \delta, \\
& z(t)=z^{0}+\frac{\beta t^{\beta-1}(1-\alpha)}{M(\alpha)} f_{3}(x, y, z, t)+\frac{\alpha \beta}{M(\alpha)} \int_{0}^{t} \delta^{\beta-1} f_{3}(x, y, z, \delta) d \delta .
\end{aligned}
$$

The following is presented at $t_{n+1}$ :

$$
\begin{aligned}
& x^{n+1}=x^{0}+\frac{\beta t^{\beta-1}(1-\alpha)}{M(\alpha)} f_{1}\left(x^{n}, y^{n}, z^{n}, t_{n}\right)+\frac{\alpha \beta}{M(\alpha)} \int_{0}^{t_{n+1}} \delta^{\beta-1} f_{1}(x, y, z, \delta) d \delta, \\
& y^{n+1}=y^{0}+\frac{\beta t^{\beta-1}(1-\alpha)}{M(\alpha)} f_{2}\left(x^{n}, y^{n}, z^{n}, t_{n}\right)+\frac{\alpha \beta}{M(\alpha)} \int_{0}^{t_{n+1}} \delta^{\beta-1} f_{2}(x, y, z, \delta) d \delta, \\
& z^{n+1}=z^{0}+\frac{\beta t^{\beta-1}(1-\alpha)}{M(\alpha)} f_{3}\left(x^{n}, y^{n}, z^{n}, t_{n}\right)+\frac{\alpha \beta}{M(\alpha)} \int_{0}^{t_{n+1}} \delta^{\beta-1} f_{3}(x, y, z, \delta) d \delta .
\end{aligned}
$$

Further, we have the following:

$$
\begin{aligned}
x^{n+1} & =x^{0}+\frac{\beta t^{\beta-1}(1-\alpha)}{M(\alpha)} f_{1}\left(x^{n}, y^{n}, z^{n}, t_{n}\right)-\frac{\beta t_{n-1}^{\beta-1}(1-\alpha)}{M(\alpha)} f_{1}\left(x^{n-1}, y^{n-1}, z^{n-1}, t_{n-1}\right) \\
& +\frac{\alpha \beta}{M(\alpha)} \int_{t_{n}}^{t_{n+1}} \delta^{\beta-1} f_{1}(x, y, z, \delta) d \delta, \\
y^{n+1} & =y^{0}+\frac{\beta t^{\beta-1}(1-\alpha)}{M(\alpha)} f_{2}\left(x^{n}, y^{n}, z^{n}, t_{n}\right)-\frac{\beta t_{n-1}^{\beta-1}(1-\alpha)}{M(\alpha)} f_{2}\left(x^{n-1}, y^{n-1}, z^{n-1}, t_{n-1}\right) \\
& +\frac{\alpha \beta}{M(\alpha)} \int_{t_{n}}^{t_{n+1}} \delta^{\beta-1} f_{2}(x, y, z, \delta) d \delta, \\
z^{n+1} & =z^{0}+\frac{\beta t^{\beta-1}(1-\alpha)}{M(\alpha)} f_{3}\left(x^{n}, y^{n}, z^{n}, t_{n}\right)-\frac{\beta t_{n-1}^{\beta-1}(1-\alpha)}{M(\alpha)} f_{3}\left(x^{n-1}, y^{n-1}, z^{n-1}, t_{n-1}\right) \\
& +\frac{\alpha \beta}{M(\alpha)} \int_{t_{n}}^{t_{n+1}} \delta^{\beta-1} f_{3}(x, y, z, \delta) d \delta .
\end{aligned}
$$




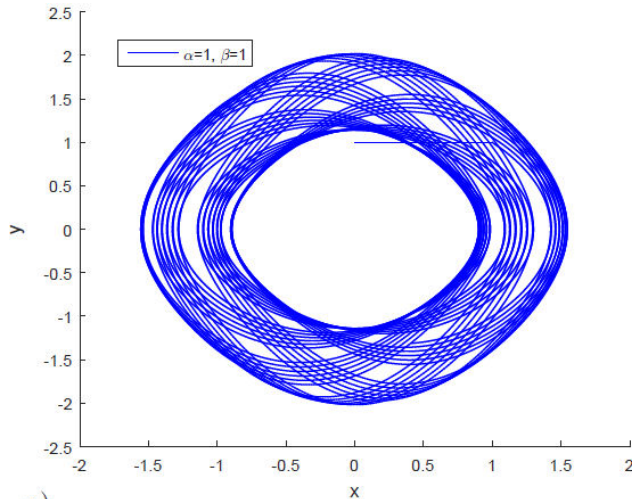

a)

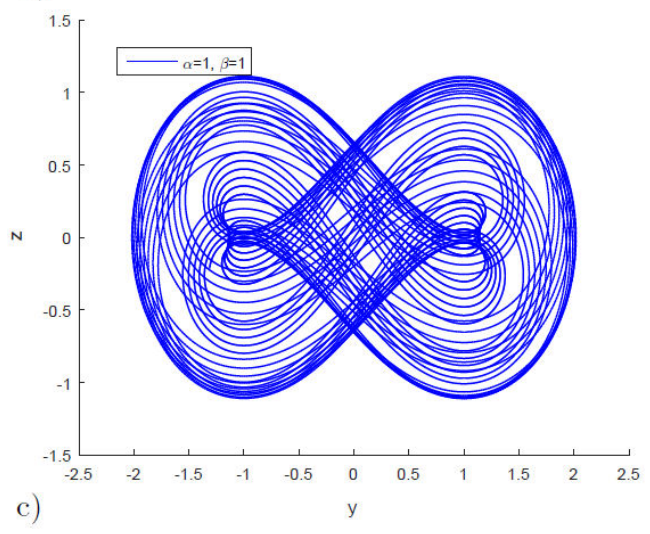

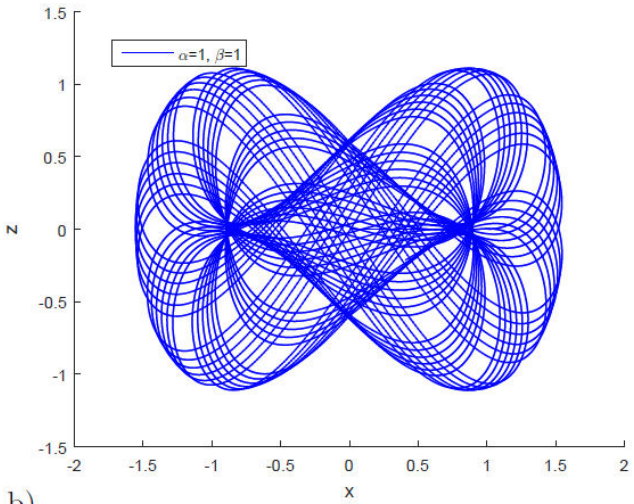

b)

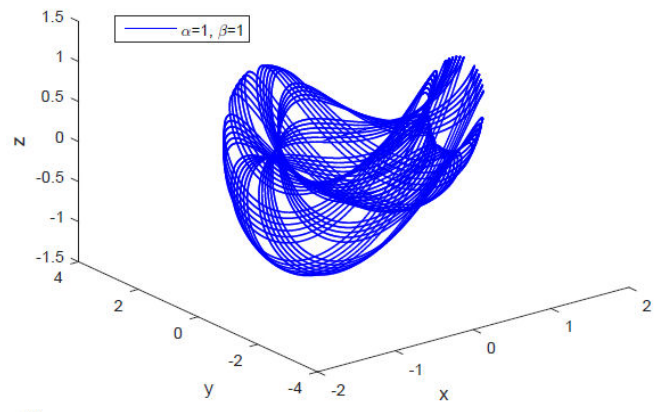

d)

FIGURE 5. FF-CF model results, when $\alpha=1, \beta=1$, where (a-d) show different phase planes.
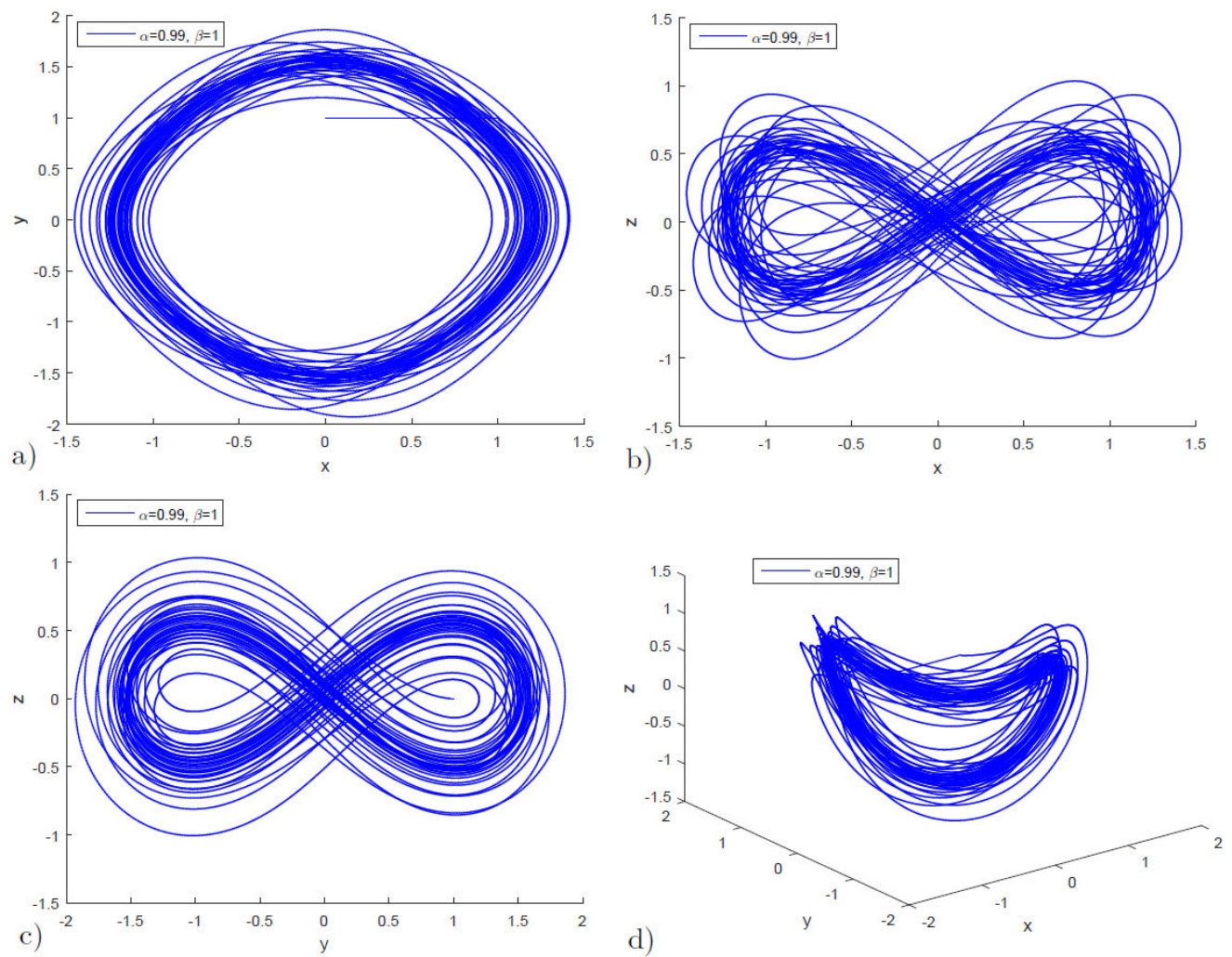

d)

FIGURE 6. FF-CF model results, when $\alpha=0.99, \beta=1$, where (a-d) show different phase planes. 

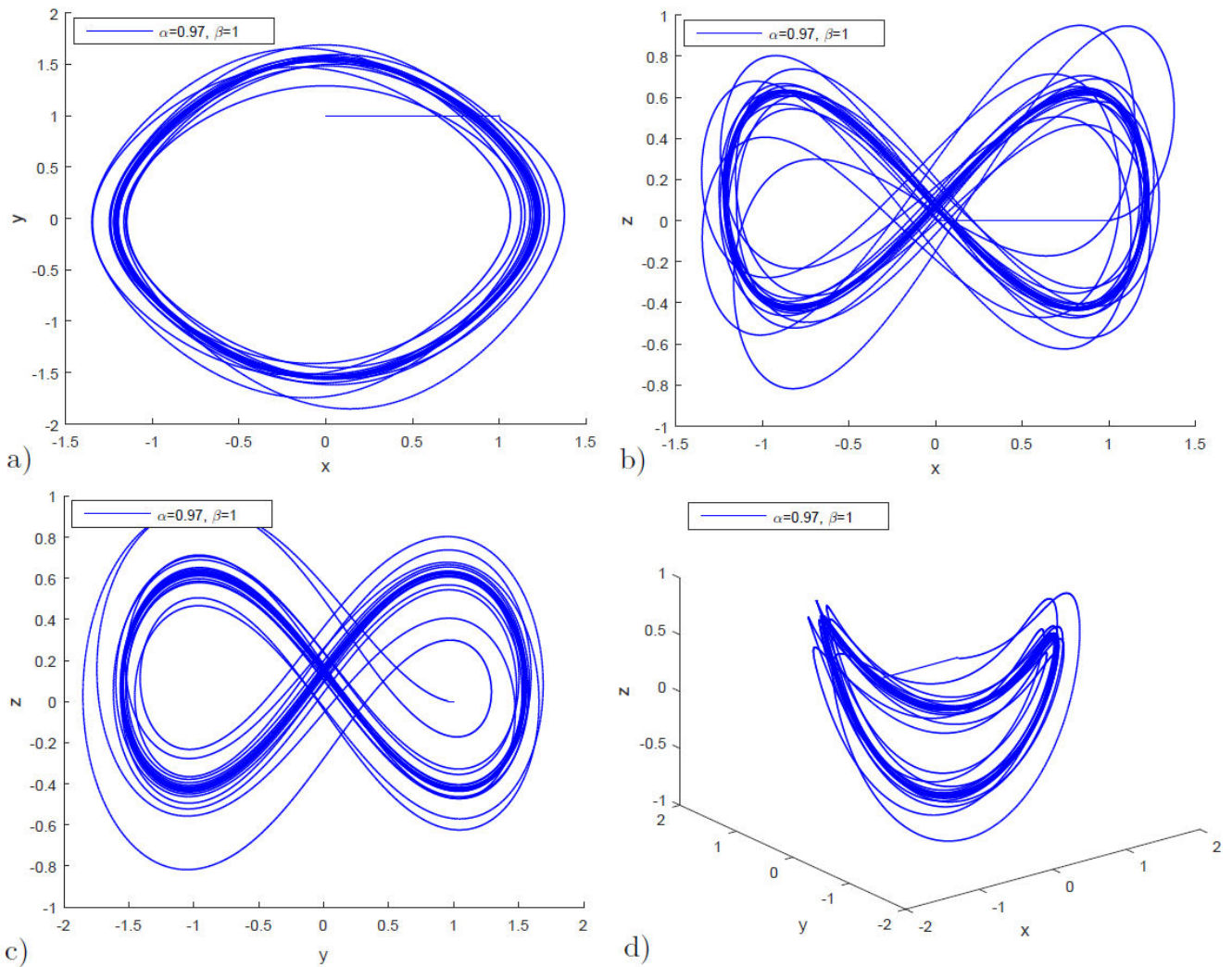

FIGURE 7. FF-CF model results, when $\alpha=0.97, \beta=1$, where (a-d) show different phase planes.
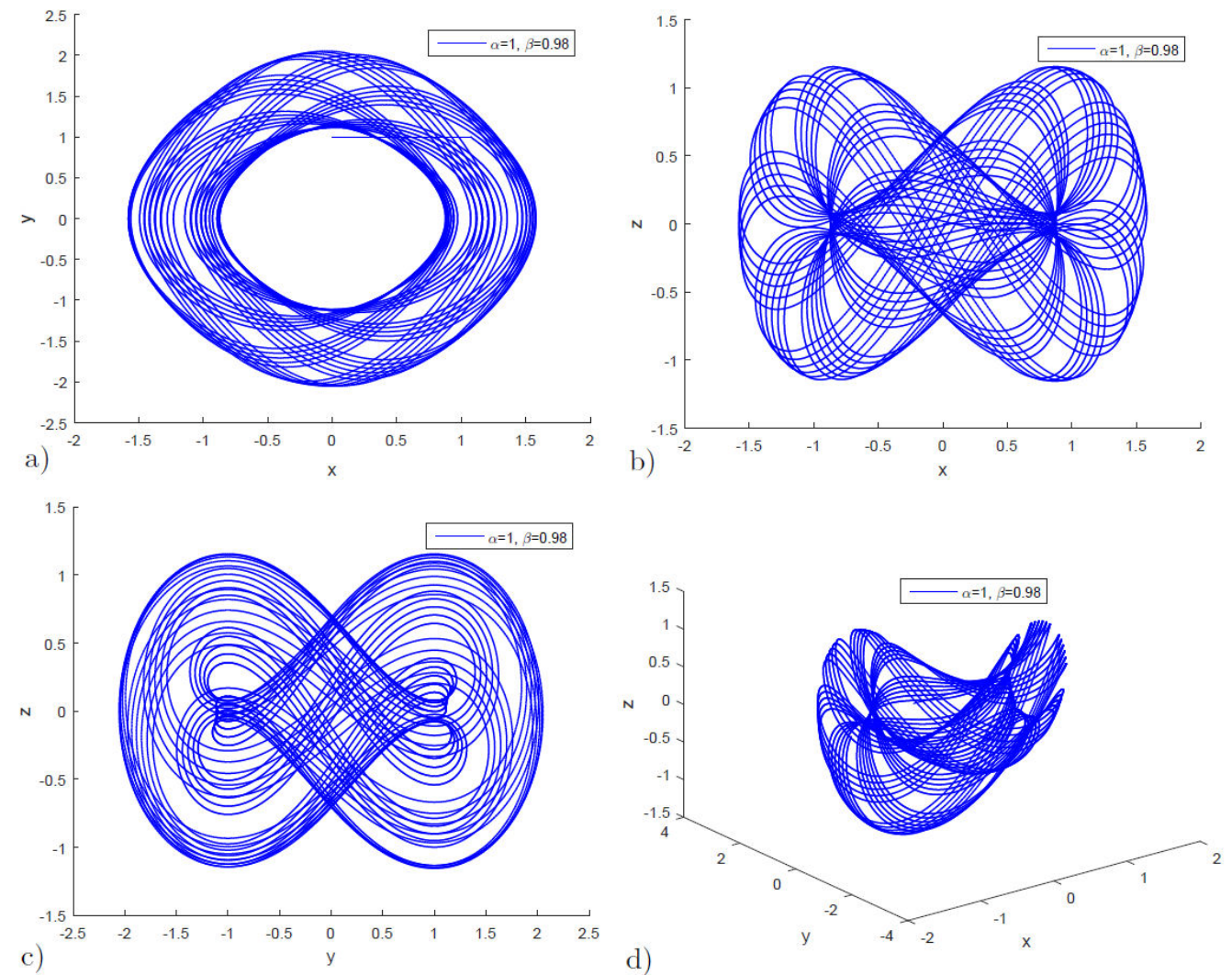

FIGURE 8. FF-CF model results, when $\alpha=1, \beta=0.98$, where (a-d) show different phase planes. 

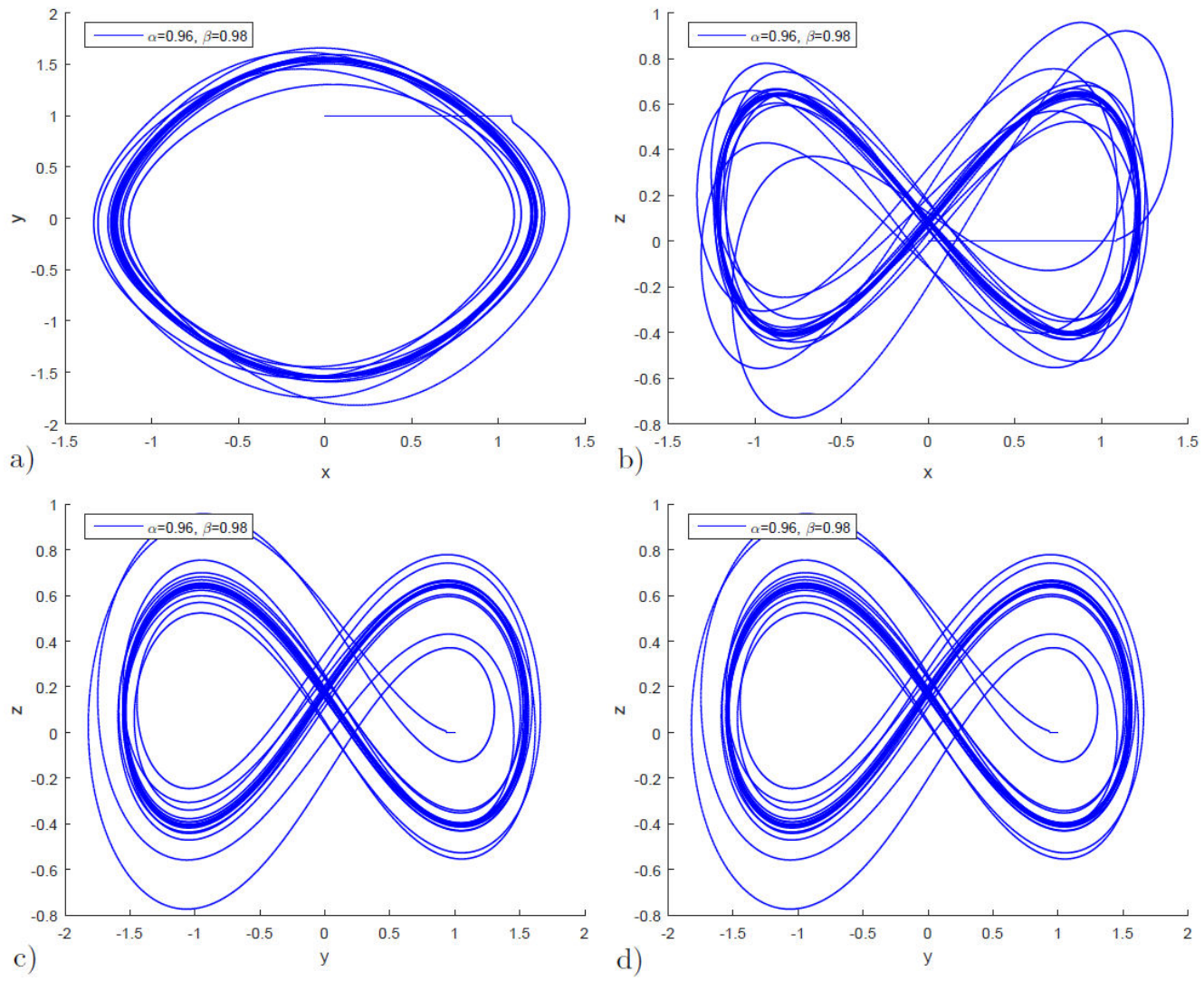

FIGURE 9. FF-CF model results, when $\alpha=0.98, \beta=0.98$, where (a-d) show different phase planes.
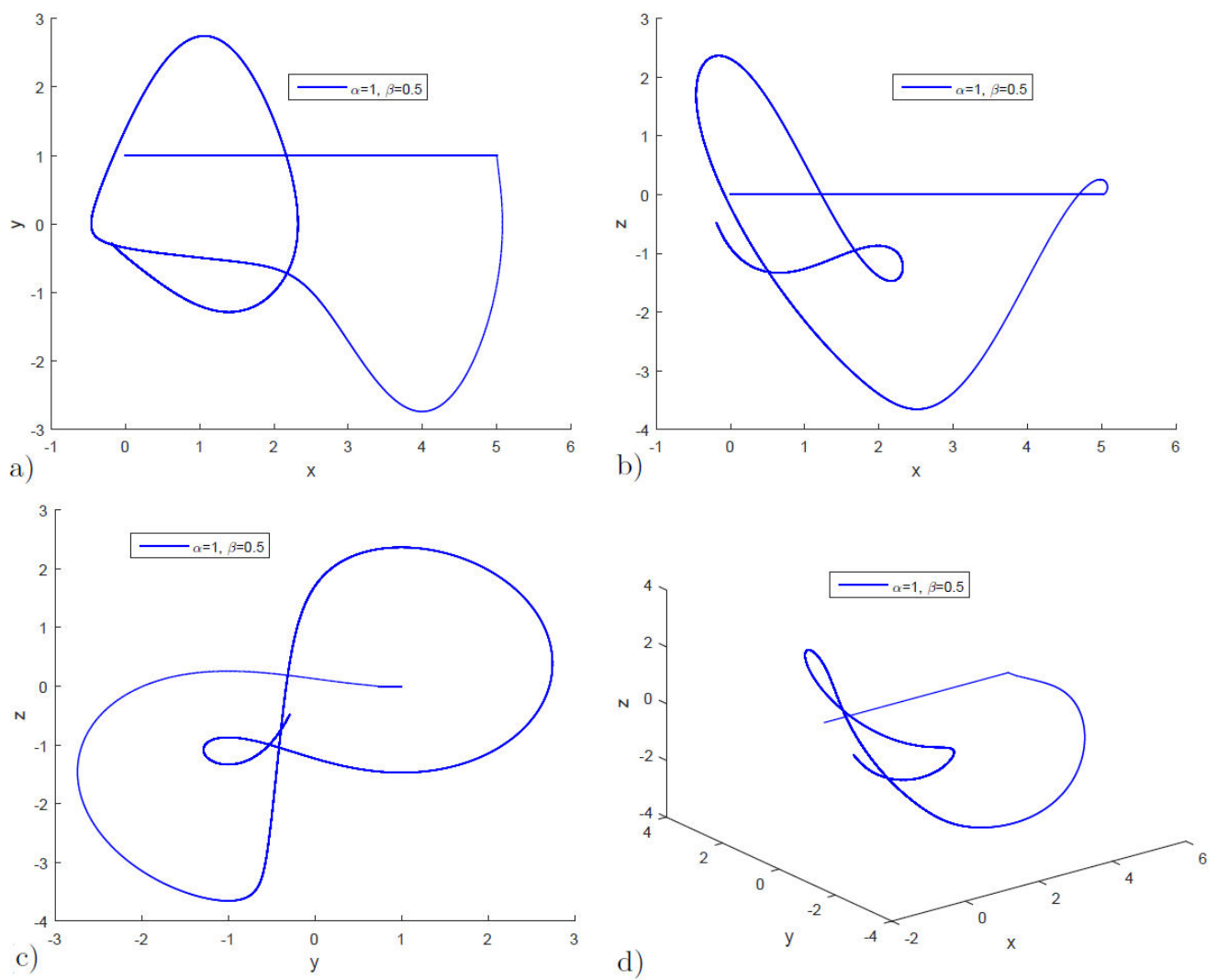

FIGURE 10. FF-CF model results, when $\alpha=1, \beta=0.5$, where (a-d) show different phase planes. 
The idea of Lagrange polynomial is used to obtain the following result:

$$
\begin{aligned}
x^{n+1} & =x^{0}+\frac{\beta t_{n}^{\beta-1}(1-\alpha)}{M(\alpha)} f_{1}\left(x^{n}, y^{n}, z^{n}, t_{n}\right)-\frac{\beta t_{n-1}^{\beta-1}(1-\alpha)}{M(\alpha)} f_{1}\left(x^{n-1}, y^{n-1}, z^{n-1}, t_{n-1}\right) \\
& +\frac{\alpha \beta}{M(\alpha)}\left[\frac{3 h}{2} t_{n}^{\beta-1} f_{1}\left(x^{n}, y^{n}, z_{2}^{n}, t_{n}\right)-\frac{h}{2} t_{n-1}^{\beta-1} f_{1}\left(x^{n-1}, y^{n-1}, z^{n-1}, t_{n-1}\right)\right], \\
y^{n+1} & =y^{0}+\frac{\beta t_{n}^{\beta-1}(1-\alpha)}{M(\alpha)} f_{2}\left(x^{n}, y^{n}, z^{n}, t_{n}\right)-\frac{\beta t_{n-1}^{\beta-1}(1-\alpha)}{M(\alpha)} f_{2}\left(x^{n-1}, y^{n-1}, z^{n-1}, t_{n-1}\right) \\
& +\frac{\alpha \beta}{M(\alpha)}\left[\frac{3 h}{2} t_{n}^{\beta-1} f_{1}\left(x^{n}, y^{n}, z_{2}^{n}, t_{n}\right)-\frac{h}{2} t_{n-1}^{\beta-1} f_{2}\left(x^{n-1}, y^{n-1}, z^{n-1}, t_{n-1}\right)\right], \\
z^{n+1} & =z^{0}+\frac{\beta t_{n}^{\beta-1}(1-\alpha)}{M(\alpha)} f_{3}\left(x^{n}, y^{n}, z^{n}, t_{n}\right)-\frac{\beta t_{n-1}^{\beta-1}(1-\alpha)}{M(\alpha)} f_{1}\left(x^{n-1}, y^{n-1}, z^{n-1}, t_{n-1}\right) \\
& +\frac{\alpha \beta}{M(\alpha)}\left[\frac{3 h}{2} t_{n}^{\beta-1} f_{3}\left(x^{n}, y^{n}, z_{2}^{n}, t_{n}\right)-\frac{h}{2} t_{n-1}^{\beta-1} f_{3}\left(x^{n-1}, y^{n-1}, z^{n-1}, t_{n-1}\right)\right] .
\end{aligned}
$$

Further, we obtain the following,

$$
\begin{aligned}
& x^{n+1}=x^{0}+\beta t_{n}^{\beta-1}\left(\frac{1-\alpha}{M(\alpha)}+\frac{3 \alpha h}{2 M(\alpha)}\right) f_{1}\left(x^{n}, y^{n}, z^{n}, t_{n}\right)-\beta t_{n-1}^{\beta-1}\left(\frac{1-\alpha}{M(\alpha)}+\frac{\alpha h}{2 M(\alpha)}\right) f_{1}\left(x^{n-1}, y^{n-1}, z^{n-1}, t_{n-1}\right), \\
& y^{n+1}=y^{0}+\beta t_{n}^{\beta-1}\left(\frac{1-\alpha}{M(\alpha)}+\frac{3 \alpha h}{2 M(\alpha)}\right) f_{2}\left(x^{n}, y^{n}, z^{n}, t_{n}\right)-\beta t_{n-1}^{\beta-1}\left(\frac{1-\alpha}{M(\alpha)}+\frac{\alpha h}{2 M(\alpha)}\right) f_{2}\left(x^{n-1}, y^{n-1}, z^{n-1}, t_{n-1}\right), \\
& z^{n+1}=z^{0}+\beta t_{n}^{\beta-1}\left(\frac{1-\alpha}{M(\alpha)}+\frac{3 \alpha h}{2 M(\alpha)}\right) f_{3}\left(x^{n}, y^{n}, z^{n}, t_{n}\right)-\beta t_{n-1}^{\beta-1}\left(\frac{1-\alpha}{M(\alpha)}+\frac{\alpha h}{2 M(\alpha)}\right) f_{3}\left(x^{n-1}, y^{n-1}, z^{n-1}, t_{n-1}\right) .
\end{aligned}
$$

Further in next subsection, we use the FF model and present a numerical procedure in order to obtain their solution numerically. We provide the details of the scheme in the below subsection.

\subsection{Numerical solution of FF-AB model}

In order to have a numerical procedure to handle the FF model in Atangana-Baleanu (AB) operator sense, the model shown in (8) can be re-written using the following way:

$$
\begin{aligned}
& { }^{A B R} D_{0, t}^{\alpha}(x(t))=\beta t^{\beta-1} f_{1}(x, y, z, t), \\
& { }^{A B R} D_{0, t}^{\alpha}(y(t))=\beta t^{\beta-1} f_{2}(x, y, z, t), \\
& { }^{A B R} D_{0, t}^{\alpha}(z(t))=\beta t^{\beta-1} f_{3}(x, y, z, t) .
\end{aligned}
$$

The following is presented based on Atangana-Baleanu (AB) integral,

$$
\begin{aligned}
& x(t)=x(0)+\frac{\beta t^{\beta-1}(1-\alpha)}{A B(\alpha)} f_{1}(x, y, z, t)+\frac{\alpha \beta}{A B(\alpha) \Gamma(\alpha)} \int_{0}^{t} \delta^{\beta-1}(t-\delta)^{\alpha-1} f_{1}(x, y, z, \delta) d \delta, \\
& y(t)=y(0)+\frac{\beta t^{\beta-1}(1-\alpha)}{A B(\alpha)} f_{2}(x, y, z, t)+\frac{\alpha \beta}{A B(\alpha) \Gamma(\alpha)} \int_{0}^{t} \delta^{\beta-1}(t-\delta)^{\alpha-1} f_{2}(x, y, z, \delta) d \delta, \\
& z(t)=z(0)+\frac{\beta t^{\beta-1}(1-\alpha)}{A B(\alpha)} f_{3}(x, y, z, t)+\frac{\alpha \beta}{A B(\alpha) \Gamma(\alpha)} \int_{0}^{t} \delta^{\beta-1}(t-\delta)^{\alpha-1} f_{3}(x, y, z, \delta) d \delta .
\end{aligned}
$$


Further, at $t_{n+1}$, we get,

$$
\begin{aligned}
& x^{n+1}=x^{0}+\frac{\beta t_{n}^{\beta-1}(1-\alpha}{A B(\alpha)} f_{1}\left(x^{n}, y^{n}, z^{n}, t_{n}\right)+\frac{\alpha \beta}{A B(\alpha) \Gamma(\alpha)} \int_{0}^{t_{n+1}} \delta^{\beta-1}\left(t_{n+1}-\delta\right)^{\alpha-1} f_{1}(x, y, z, \delta) d \delta, \\
& y^{n+1}=y^{0}+\frac{\beta t_{n}^{\beta-1}(1-\alpha}{A B(\alpha)} f_{2}\left(x^{n}, y^{n}, z^{n}, t_{n}\right)+\frac{\alpha \beta}{A B(\alpha) \Gamma(\alpha)} \int_{0}^{t_{n+1}} \delta^{\beta-1}\left(t_{n+1}-\delta\right)^{\alpha-1} f_{2}(x, y, z, \delta) d \delta \\
& z^{n+1}=z^{0}+\frac{\beta t_{n}^{\beta-1}(1-\alpha}{A B(\alpha)} f_{3}\left(x^{n}, y^{n}, z^{n}, t_{n}\right)+\frac{\alpha \beta}{A B(\alpha) \Gamma(\alpha)} \int_{0}^{t_{n+1}} \delta^{\beta-1}\left(t_{n+1}-\delta\right)^{\alpha-1} f_{3}(x, y, z, \delta) d \delta .
\end{aligned}
$$

We obtain the following after simplifying the integral in the above equations:

$$
\begin{aligned}
& x^{n+1}=x^{0}+\frac{\beta t_{n}^{\beta-1}(1-\alpha)}{A B(\alpha)} f_{1}\left(x^{n}, y^{n}, z^{n}, t_{n}\right)+\frac{\alpha \beta}{A B(\alpha) \Gamma(\alpha)} \sum_{j=0}^{n} \int_{t_{j}}^{t_{j+1}} \delta^{\beta-1}\left(t_{n+1}-\delta\right)^{\alpha-1} f_{1}(x, y, z, \delta) d \delta, \\
& y^{n+1}=y^{0}+\frac{\beta t_{n}^{\beta-1}(1-\alpha)}{A B(\alpha)} f_{2}\left(x^{n}, y^{n}, z^{n}, t_{n}\right)+\frac{\alpha \beta}{A B(\alpha) \Gamma(\alpha)} \sum_{j=0}^{n} \int_{t_{j}}^{t_{j+1}} \delta^{\beta-1}\left(t_{n+1}-\delta\right)^{\alpha-1} f_{2}(x, y, z, \delta) d \delta \\
& z^{n+1}=z^{0}+\frac{\beta t_{n}^{\beta-1}(1-\alpha)}{A B(\alpha)} f_{3}\left(x^{n}, y^{n}, z^{n}, t_{n}\right)+\frac{\alpha \beta}{A B(\alpha) \Gamma(\alpha)} \sum_{j=0}^{n} \int_{t_{j}}^{t_{j+1}} \delta^{\beta-1}\left(t_{n+1}-\delta\right)^{\alpha-1} f_{3}(x, y, z, \delta) d \delta .
\end{aligned}
$$

Now, approximating the expressions in (26) given by $\delta^{\beta-1} f_{1}(x, y, z, \delta), \delta^{\beta-1} f_{2}(x, y, z, \delta)$ and $\delta^{\beta-1} f_{3}(x, y, z, \delta)$ in the given interval $\left[t_{j}, t_{j+1}\right]$, the following numerical scheme is presented,

$$
\begin{aligned}
x^{n+1} & =x^{0}+\frac{\beta t_{n}^{\beta-1}(1-\alpha)}{A B(\alpha)} f_{1}\left(x^{n}, y^{n}, z^{n}, t_{n}\right)+\frac{\beta(\Delta t)^{\alpha}}{A B(\alpha) \Gamma(\alpha+2)} \\
& \times \sum_{j=1}^{n}\left[t_{j}^{\beta-1} f_{1}\left(x^{j}, y^{j}, z^{j}, t_{j}\right) a_{n, j}-t_{j-1}^{\beta-1} f_{1}\left(x^{j-1}, y^{j-1}, z^{j-1}, t_{j-1}\right) b_{n, j}\right], \\
y^{n+1} & =y^{0}+\frac{\beta t_{n}^{\beta-1}(1-\alpha)}{A B(\alpha)} f_{2}\left(x^{n}, y^{n}, z^{n}, t_{n}\right)+\frac{\beta(\Delta t)^{\alpha}}{A B(\alpha) \Gamma(\alpha+2)} \\
& \times \sum_{j=1}^{n}\left[t_{j}^{\beta-1} f_{2}\left(x^{j}, y^{j}, z^{j}, t_{j}\right) a_{n, j}-t_{j-1}^{\beta-1} f_{2}\left(x^{j-1}, y^{j-1}, z^{j-1}, t_{j-1}\right) b_{n, j}\right], \\
z^{n+1} & =z^{0}+\frac{\beta t_{n}^{\beta-1}(1-\alpha)}{A B(\alpha)} f_{3}\left(x^{n}, y^{n}, z^{n}, t_{n}\right)+\frac{\beta(\Delta t)^{\alpha}}{A B(\alpha) \Gamma(\alpha+2)} \\
& \times \sum_{j=1}^{n}\left[t_{j}^{\beta-1} f_{3}\left(x^{j}, y^{j}, z^{j}, t_{j}\right) a_{n, j}-t_{j-1}^{\beta-1} f_{3}\left(x^{j-1}, y^{j-1}, z^{j-1}, t_{j-1}\right) b_{n, j}\right],
\end{aligned}
$$

where

$$
\begin{aligned}
& a_{n, j}=\left((n+1-j)^{\alpha}(n-j+2+\alpha)-(n-j)^{\alpha}(n-j+2+2 \alpha)\right), \\
& b_{n, j}=\left((n-j+1)^{\alpha+1}-(n-j)^{\alpha}(n-j+1+\alpha)\right),
\end{aligned}
$$

where $n=0$ to $N$ and $j=1$ to $n$. 

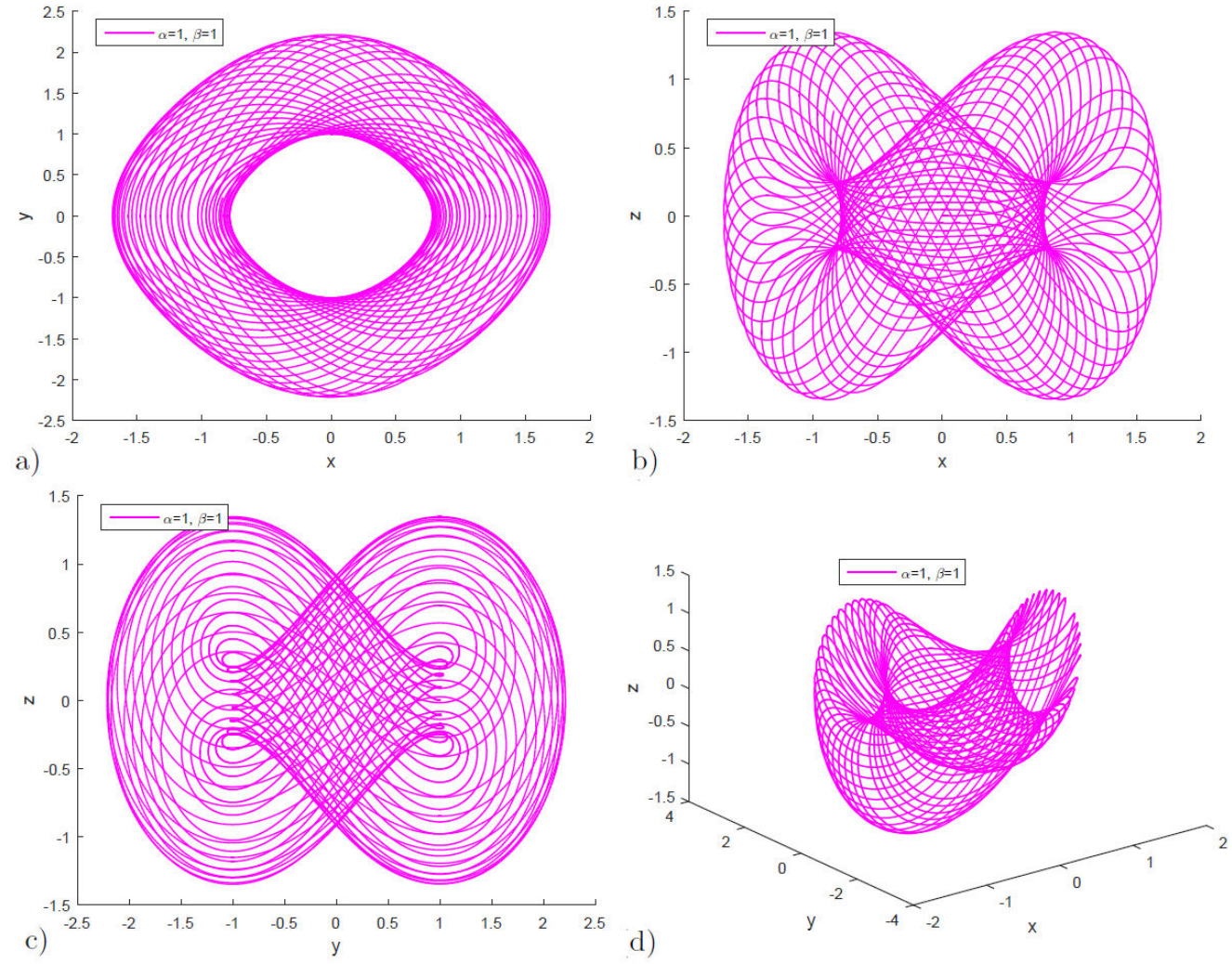

FIGURE 11. FF-AB model results, when $\alpha=1, \beta=1$, where (a-d) show different phase planes.
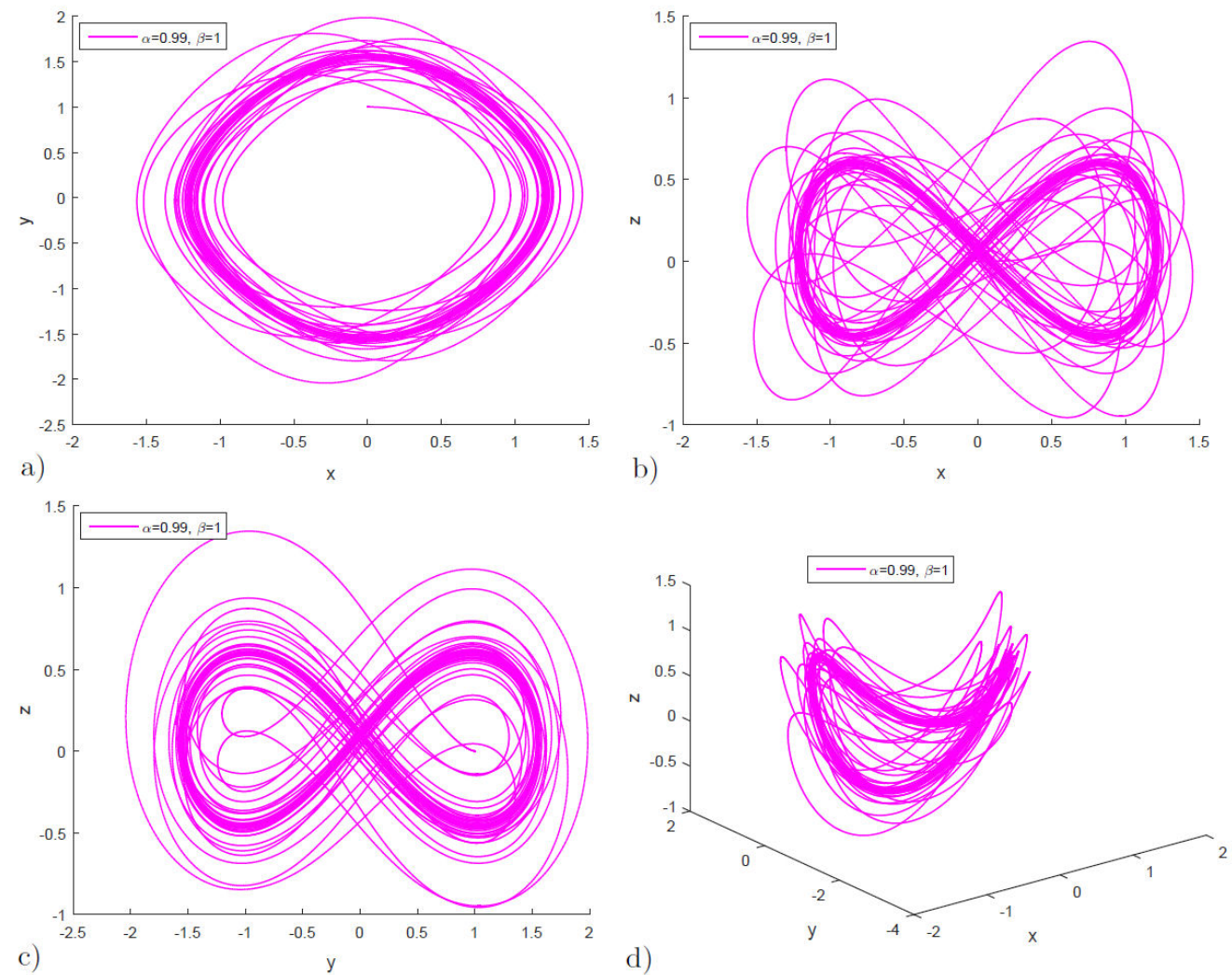

FIGURE 12. FF-AB model results, when $\alpha=0.99, \beta=1$, where (a-d) show different phase planes. 


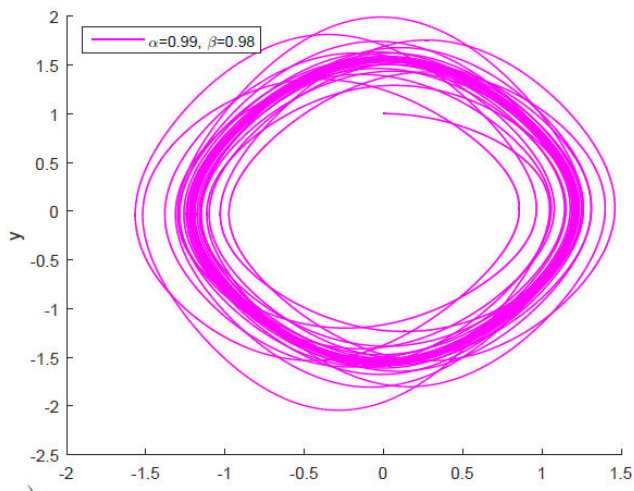

a)
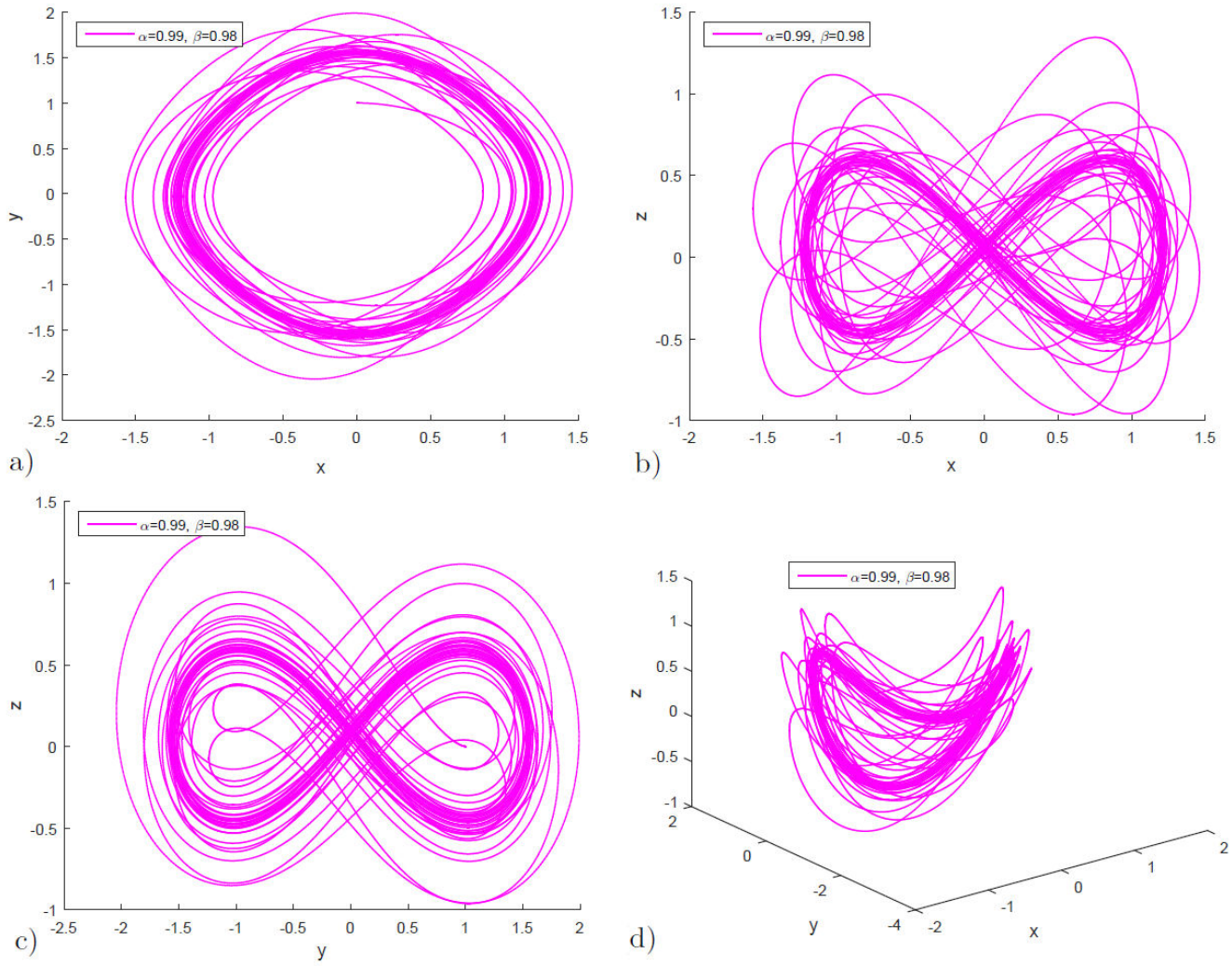

FIGURE 13. FF-AB model results, when $\alpha=0.99, \beta=0.98$, where (a-d) show different phase planes.
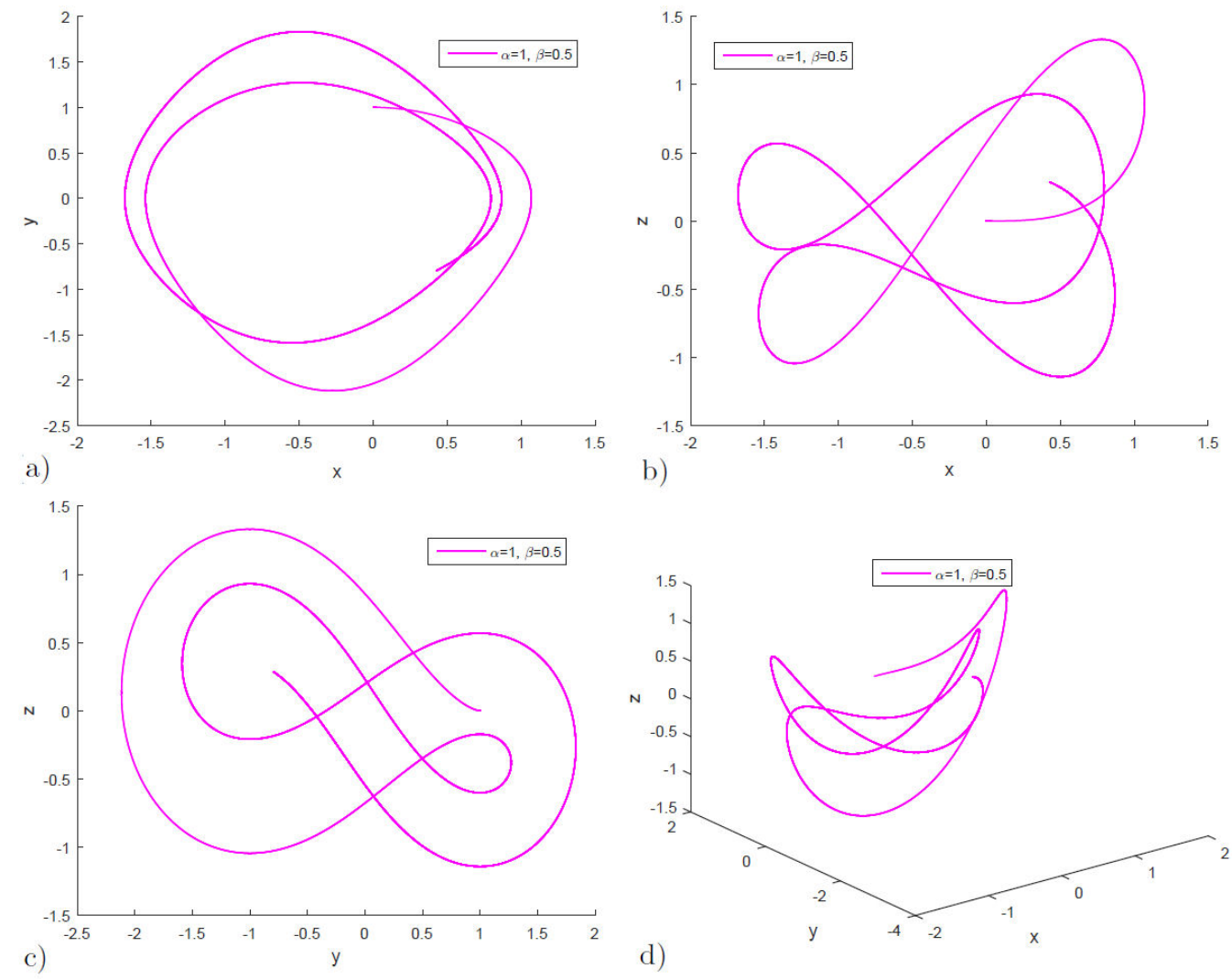

d)

FIGURE 14. FF-AB model results, when $\alpha=1, \beta=0.5$, where (a-d) show different phase planes. 


\section{Discussion}

We studied above the detailed algorithms for the solution of FF models numerically using the power law, exponential decay, and the Mittag-Leffler cases. For each operator, the scheme has been presented in a detailed manner. We use the numerical algorithm shown above in order to obtain the solution of the chaotic model in graphical form. Using the scheme presented in (16) for the FF system in Caputo sense, we used the scheme, and the corresponding graphical results are shown in Figs. 1-4 with different fractal and fractional orders. We see, for different orders of fractal and fractional, the model behaves chaotically. We also utilized a different set of fractal and fractional orders and obtained interesting chaotic results. For the model formulated in FF-CF derivative, we considered the suggested scheme (22), and the solution of the model has been shown graphically in Figs. 5-10, with arbitrary orders of fractal and fractional orders. One can observe that different combinations of the suggested operators provide useful numerical results. Further, the procedure given in (27) is used for the numerical results of the fractalfractional Atangana-Baleanu model with arbitrary order of fractal and fractional order parameters values and obtain the Figs. 11-14. From all these results, it can be concluded that the chaotic model provides different behaviors for the suggested values of the fractal and fractional orders. Using these fractal-fractional operators, many chaotic results and hidden attractors can be studied effectively. These operators can be applied to other nonlinear problems arising from physical sciences, social sciences, and engineering areas where one can find the best possible results for their proposed problems.

\section{Conclusion}

We presented a chaotic model in FF operators where the fractional operators were considered in Caputo, $\mathrm{CF}$, and $\mathrm{AB}$ sense. We presented briefly the simulation of the chaotic model by using novel numerical approaches. We obtained the numerical results presented for the solution of the fractalfractional model in the sense of three different fractalfractional operators. We used the initial value for $t=0.1$ and the step size $h=0.01$. We presented the graphical results for each operator by selecting some suitable values for the fractal and fractional orders. We see that for each value of the fractal and fractional order, different chaotic behaviors of the model are observed. It is observed that by changing the values of the fractal and fractional orders, some new chaotic results are obtained graphically. This new analysis of the FF model in different operators bring new doors of investigations for scientists and researchers working in modeling electrical circuits theory. The results reveal that the fractalfractional operators and their application to a chaotic model provide useful results that cannot be seen for an ordinary operator. These operators can be beneficial in modeling other engineering fields and sciences related areas.

\section{Conflict of interest}

No conflict of interest exists regarding the publications of this work.

\section{Acknowledgment}

The authors extend their appreciation to the Deanship of Scientific Research at King Khalid University, Abha, Saudi Arabia for funding this work through general research groups program under grant number G.R.P-150/42.
1. B. Wang, S. Zhou, X. Zheng, Image watermarking using chaotic map and dna coding, Optik. 126 (2015) 4846-4851. $10.1016 / j . i j l e o .2015 .09 .217$

2. S. Çiçek, A. Ferikoğlu, and I. Pehlivan, A new $3 d$ chaotic system: dynamical analysis, electronic circuit design, active control synchronization and chaotic masking communication application Optik-International Journal for Light and Electron Optics. 127(8) (2016) 4024-4030.10.1016/j.ijleo. 2016.01 .069

3. C. K Volos, I.M. Kyprianidis, and I. N. Stouboulos, A chaotic path planning generator for autonomous mobile robots, Robotics and Autonomous Systems. 60(4) (2012) 651-656. https://doi.org/10.1016/j.robot.2012.01. 001

4. C. Xu, M. Liao, P. Li, Q. Xiao, and S. Yuan, Control strategy for a fractional-order chaotic financial model, Complexity. 2019, 2019. $10.1016 / j$.chaos.2019.04.020
5. O. Atan, Synchronisation and circuit model of fractionalorder chaotic systems with time-delay, IFAC-PapersOnLine, 49 (29) (2016) 68-72. https://doi.org/10.1177/ 1687814019866540

6. A. Atangana and S. Qureshi, Modeling attractors of chaotic dynamical systems with fractal-fractional operators, Chaos, Solitons \& Fractals. 123 (2019) 320-337. DOI:10.1016/j. chaos.2019.04.020

7. M. A. Khan, The dynamics of a new chaotic system through the caputo-fabrizio and atanagan-baleanu fractional operators, Advances in Mechanical Engineering, 11(7):1687814019866540, 2019. https://doi.org/10. $1177 / 1687814019866540$

8. A. Atangana, Fractional discretization: The african's tortoise walk, Chaos, Solitons \& Fractals, 130:109399, 2020. https: //doi.org/10.1016/j.chaos.2019.109399 
9. B. Ghanbari and A. Atangana. A new application of fractional atangana-baleanu derivatives: Designing abc-fractional masks in image processing, Physica A: Statistical Mechanics and its Applications, 2019.

10. M. A. Khan, O. Kolebaje, A. Yildirim, S. Ullah, P. Kumam, and P. Thounthong, Fractional investigations of zoonotic visceral leishmaniasis disease with singular and non-singular kernel, The European Physical Journal Plus. 134 (2019) 481. https : //doi.org/10.1140/epjp/i2019-12861-1

11. R. Jan, M. A. Khan, P. Kumam, and P. Thounthong, Modeling the transmission of dengue infection through fractional derivatives, Chaos, Solitons \& Fractals, 127 (2016) 189-216. $10.1016 / j$.chaos.2019.07.002

12. M. A. Khan and F. Gómez-Aguilar, Tuberculosis model with relapse via fractional conformable derivative with power law, Mathematical Methods in the Applied Sciences. 2019.

13. S. Ullah, M. A. Khan, M. Farooq, Z. Hammouch, and D. Baleanu, A fractional model for the dynamics of tuberculosis infection using caputo-fabrizio derivative, Discrete \& Continuous Dynamical Systems-S, 29 (2019) 11-27.doi:10.3934/ dcdss.2020057

14. S. Ullah, M. A. Khan, and M. Farooq, A fractional model for the dynamics of $t b$ virus, Chaos, Solitons \& Fractals. 116 (2018) 63-71.

15. A. Atangana and S. İğret Araz. Fractional stochastic modelling illustration with modified chua attractor, The European Physical Journal Plus. 134(4) (2019) 160. https://doi.org/ 10.1140/epjp/i2019-12565-6

16. S. Ullah, M. A. Khan, and M. Farooq, Modeling and analysis of the fractional hbv model with atanganabaleanu derivative, The European Physical Journal Plus. 133(8) (2018) 313. https: / / doi .org/10.1140/epjp/ i2018-12120-1

17. A. Atangana and M. A. Khan, Validity of fractal derivative to capturing chaotic attractors, Chaos, Solitons \& Fractals. 126 (2019)50-59.10.1016/j.chaos.2019.06.002

18. W. Sayed, AG. Radwan, Two-dimensional rotation of chaotic attractors: Demonstrative examples and fpga realization, Circuits, Systems, and Signal Processing. 38(10) (2019) 4890-4903. https://doi.org/10.1007/ s00034-019-01096-z

19. M. S. Semary, A. Radwan, Realization of fractional-order capacitor based on passive symmetric network, Journal of advanced research. 18 (2019) 147-159. https://doi.org/ $10.1016 / j$.jare.2019.02.004

20. A. J. Abd El-Maksoud, A. A Abd El-Kader, Fpga implementation of sound encryption system based on fractional-order chaotic systems, Microelectronics Journal. 90 (2019) 323-335. https://doi.org/10.1016/j.mejo.2019.05. 005

21. J. F. Gomez-Aguilar, Chaos and multiple attractors in a fractal-fractional Shinriki's oscillator model, Physica A: Statistical Mechanics and its Applications. 539 ( 2020) 122918. https://doi.org/10.1016/j.physa. 2019.122918

22. J. F. Gomez-Aguilar, Multiple attractors and periodicity on the Vallis model for El Niño/La Niña-Southern oscillation model, Journal of Atmospheric and Solar-Terrestrial Physics. 197 (2020) 105172. https://doi.org/10.1016/j. jastp.2019.105172

23. B. Ghanbari, J. F. Gómez-Aguilar, Analysis of two avian influenza epidemic models involving fractal-fractional derivatives with power and Mittag-Leffler memories. Chaos: An Interdisciplinary Journal of Nonlinear Science. 29 (2019) 123113. https://doi.org/10.1063/1.5117285

24. J. F. Gómez-Aguilar, T. Cordova-Fraga, T. Abdeljawad, A. Khan, H. Khan. Analysis of fractal-fractional malaria transmission model, Fractals. (2020) 7:2040041. https://doi. org/10.1142/S0218348X20400411

25. J. F. Gomez-Aguilar, New chaotic attractors: Application of fractal-fractional differentiation and integration, Mathematical Methods in the Applied Sciences, 44 ( 2021) 3036-65. https://doi.org/10.1002/mma.6432

26. K. M. Saad, M. Alqhtani, J. F. Gómez-Aguilar, Fractalfractional study of the hepatitis $C$ virus infection model. Results in Physics. 2020 Dec 1;19:103555. https://doi.org/ $10.1016 / j . r i n p .2020 .103555$

27. C. J. Zúñiga-Aguilar, J. F. Gómez-Aguilar, H. M. RomeroUgalde, H. Jahanshahi, F. E. Alsaadi, Fractal-fractional neuroadaptive method for system identification. Engineering with Computers. 22 (2021) 1-24.

28. S. Kumar, A new analytical modelling for fractional telegraph equation via Laplace transform, Applied Mathematical Modelling. 38 ( 2014) 3154-63. https://doi.org/10. $1016 / j . a p m .2013 .11 .035$

29. B. Ghanbari, S. Kumar, R. Kumar, A study of behaviour for immune and tumor cells in immunogenetic tumour model with non-singular fractional derivative, Chaos, Solitons \& Fractals. 133 (2020) 109619. DOI: $10.1016 /$ j.chaos. 2020. 109619

30. E. F. Goufo, S. Kumar, S. B. Mugisha, Similarities in a fifth-order evolution equation with and with no singular kerne Chaos, Solitons \& Fractals. 130 (2020) 109467. DOI: 10. $1016 / j . c h a o s .2019 .109467$

31. S. Kumar, R. Kumar, R.P. Agarwal, B. Samet, A study of fractional Lotka-Volterra population model using Haar wavelet and Adams-Bashforth Moulton methods, Mathematical Methods in the Applied Sciences. 43 (2020) 5564-5578. https: //doi.org/10.1002/mma.6297

32. S. Kumar, S. Ghosh, B. Samet, E. F. Goufo, An analysis for heat equations arises in diffusion process using new YangAbdel-Aty-Cattani fractional operator, Mathematical Methods in Applied Sciences, 43 (2020) 6062-6080. https : / / doi. org/10.1002/mma.6347

33. P. Veeresha, D. G. Prakasha, S. Kumar, A fractional model for propagation of classical optical solitons by using nonsingular derivative, Mathematical Methods in Applied Sciences, https://doi.org/10.1002/mma.6335(2020)

34. S. Kumar, S. Ghosh, R. Kumar, M. Jleli, A fractional model for population dynamics of two interacting species by using spectral and Hermite wavelets methods, Numerical Methods for Partial Differential Equations, 37 (2021) 1652-1672. https : //doi.org/10.1002/num.22602 
35. S. Kumar, R. Kumar, M. S. Osman, B. Samet, A wavelet based numerical scheme for fractional order SEIR epidemic of measles by using Genocchi polynomials, Numerical Methods for Partial Differential Equations, 37 (2021) 1250-1268. https://doi.org/10.1002/num.22577

36. Z. Li, Z. Liu, and M. A. Khan, Fractional investigation of bank data with fractal-fractional caputo derivative, Chaos, Solitons \& Fractals. page 109528, 2019.

37. W. Wang and M. A. Khan, Analysis and numerical simulation of fractional model of bank data with fractal-fractional atangana-baleanu derivative, Journal of Computational and
Applied Mathematics. 369 (2020) 112-646. https: / /doi. org/10.1016/j.cam.2019.112646

38. A. Atangana, M. A. Khan, et al. Modeling and analysis of competition model of bank data with fractal-fractional caputofabrizio operator, Alexandria Engineering Journal, 2020. https://doi.org/10.1016/j.aej.2019.12.032

39. T. Mekkaoui, Z. Hammouch, F. BM Belgacem, and A. El. Abbassi, Fractional-order nonlinear systems: Chaotic dynamics, numerical simulation and circuits design, Fractional Dynamics. 343-356. Sciendo Migration, 2015. 